\title{
The miR-19b-3p/MAP2K3/STAT3 Feedback Loop Regulates Cell Proliferation and Invasion in Esophageal Squamous Cell Carcinoma
}

\section{Ying Zhang ( $\sim$ 47122404@qq.com )}

Shantou University Medical College

\section{Zhaoyong Liu}

First Hospital of Medical College of Shantou University

\section{Weiqing Lu}

First Hospital of Medical College of Shantou University

\section{Xia Yang}

Sun Yat-sen University Cancer Center

\section{Yelong Chen}

First Hospital of Medical College of Shantou University

Hu Wang

First Hospital of Medical College of Shantou University

\section{Research}

Keywords: MAP2K3, STAT3, miR-19b-3p, MDM2

Posted Date: July 23rd, 2020

DOl: https://doi.org/10.21203/rs.3.rs-46772/v1

License: (9) (i) This work is licensed under a Creative Commons Attribution 4.0 International License. Read Full License 


\section{Abstract}

Background Esophageal squamous cell carcinoma (ESCC) remains one of the most refractory malignancies worldwide. MAP2K3 has been reported to play an important role in tumor progression. However, whether MAP2K3 also affects ESCC remains to be determined.

Method We used a CRISPR/Cas9 kinome screen to identify the genes related to ESCC cell survival. The MAP2K3 expression was detected in ESCC tissues by immunohistochemistry and westernblot. The function of MAP2K3 in ESCC was investigated using colony formation assay and Transwell assay in vivo and in vitro. RNA sequence was performed to verify its downstream signaling pathways. DNA binding of the gene promoter region was detected by chromatin immunoprecipitation.

Result Downregulation of MAP2K3 was found in ESCC and correlated with clinically poor survival. MAP2K3 inhibited cell proliferation and invasion via the EGFR/STAT3 signaling pathway in ESCC cells. MAP2K3 suppressed STAT3 expression and activation by interacting with MDM2 to promote the ubiquitin proteasome degradation of STAT3. Furthermore, MAP2K3 was a downstream target of miR-19b-3p, which promoted ESCC tumorigenesis. STAT3 binds to the MIR19B promoter region to increase the expression of miR-19b-3p in ESCC cells.

Conclusion In summary, our results demonstrated that the miR-19b-3p/MAP2K3/STAT3 feedback loop regulates tumorigenesis in ESCC and elucidate the potential of therapeutically targeting this pathway in ESCC.

\section{Introduction}

Esophageal cancer, especially esophageal squamous cell carcinoma (ESCC), is one of the most common primary malignant tumors in China, with a 5 -year survival of only $19 \%[1]$. Surgery supplemented by chemoradiotherapy is still the main treatment for esophageal cancer. However, unlike non-small-cell lung cancer and melanoma, the most frequent therapeutic targets in ESCC are currently undruggable and novel targets urgently needed[2, 3].

Mitogen-activated protein kinase 3 (MAP2K3), a dual specificity kinase in the MAP kinase kinase (MKK) family, is activated by MKK kinase proteins through Ser-189 and Thr-193 phosphorylation. MAP2K3 was identified as an oncogene whose depletion reduces tumor growth and improves biological response to chemotherapy[4, 5]. In ESCC, a MAP2K3 inhibitor was reported to suppress cell growth[6]. However, the role of MAP2K3 in cancer has been challenged recently. A novel loss of MAP2K3 genomic copy number was observed in breast cancer patients and the overexpression of MAP2K3 inhibited breast cancer cell proliferation by promoting cell cycle arrest[7]. Furthermore, MAP2K3 was found to promote breast cancer cell senescence. Although these findings support the notion that aberrant MAP2K3 expression is involved in several important tumorigenic molecular pathways, the expression and biological function of MAP2K3 in ESCC remains to be elucidated. 
Signal transducer and activator of transcription (STAT)3, a transcription factor that regulates cell cycle progression, apoptosis, and angiogenesis, belongs to a family of transcription factors that transduce cellular signals from a number of cytokines and soluble growth factors such as IL-6 family cytokines and interferon (IFN) [8]. STAT3 plays an important role in tumorigenesis and its activation induces expression of apoptosis inhibitors and cell-cycle regulators that participate in oncogenesis, such as vascular endothlial growth factor(VEGF), cyclin D1 and survivin [9]. STAT3 expression have also been correlated with reduced survival and poor prognosis in patients with ESCC, breast cancer and lung cancer[10]. However, the correlation between MAP2K3 and STAT3 has never been explored.

In this study, we employed a clustered regularly interspaced short palindromic repeats/associated protein 9 (CRISPR/Cas9) kinome screened and identified MAP2K3 as an ESCC tumor suppressor gene. The expression and function of MAP2K3 and its downstream signaling pathway EGFR/STAT3 were subsequently explored in ESCC. Furthermore, we also found that MAP2K3 is suppressed by miR-19b-3p, which is transcriptionally activated by STAT3 in ESCC cells. Our study is the first to demonstrate that the miR-19b-3p/MAP2K3/STAT3 feedback loop regulates ESCC tumorigenesis. Therefore, MAP2K3 may represent a predictor and potential therapeutic target for ESCC.

\section{Material And Methods}

\section{Cells and patient samples}

ESCC cells lines KYSE150, KYSE520, TE1, KYSE180, KYSE450, KYSE410 and the immortalized esophageal epithelial cell line NE1 were maintained in Dulbecco' s modified Eagle' s medium (DMEM, Gibco, NY, USA) and RPMI 1640 supplemented with $10 \%$ fetal bovine serum (FBS, Gibco) with $5 \% \mathrm{CO}_{2}$ in $37^{\circ} \mathrm{C}$. This study included 140 randomly selected patients with primary ESCC who underwent radical esophageal resection at the First Affiliated Hospital of Shantou University Medical College and Sun Yatsen University Cancer Center from 2010 to 2017. None of the patients received preoperative radiotherapy or chemotherapy. $106 / 140(75 \%)$ were male and $34 / 140$ (25\%) were female. The median age was 57.8 years (range, 37-75 years). This study was approved by the ethical review committees of the Sun Yat-sen University Cancer Center. All participants involved in our study provided written informed consent.

\section{Pooled CRISPR/Cas9 sgRNA screen and establishment of MAP2K3 knockout (KO) cell lines}

A pooled lentivirus-based plasmid library, encompassing 2925 different sgRNAs against 976 human kinase genes (3 sgRNAs per gene), was obtained from EdiGene (Beijing, China). The list of genes was obtained from the KinBase database (http://kinase.com/human/kinome/). The library was co-transfected, with a lentivirus expression system, using Fugene HD (Promega, WI, USA). Lentivirus was packaged and concentrated by using a three-plasmid system (pCMVR8.74, pcmv-vsv-g \& cas9-2a-mchy-bsd) to obtain the high-titer lentivirus wrapped in cas9-2a-mchy-bsd. Forty-eight hours after transfection, lentiviruscontaining supernatants were collected and clarified by centrifugation. After selection with puromycin for 7 days, $2 \times 10^{8}$ cells were infected ( $\mathrm{MOI}$ of 0.3 ) and then incubated for 2-3 weeks with or without drug 
treatment. Genomic DNA was extracted from transfected or control cells, and sgRNA sequences were amplified, and subjected to next generation sequencing using an Illumina HiSeq 2500 platform.

The stable knockout (KO) cell lines which were negative for all isoforms of MAP2K3 were established from KYSE520 cells using the CRISPR/Cas9 system. Briefly, sgRNA targeting the MAP2K3 gene were designed using the CRISPR Design Online Tool (https://www.genscript.com/gRNA-design-tool.html). After transfection, the puromycin-resistant cells were selected and expanded. Westernblot and Sanger sequencing were performed to confirm the knockout.

\section{Nuclear and cytoplasmic extraction and western blot analysis}

NE-PER ${ }^{T M}$ nuclear and cytoplasmic extraction reagents (Thermo Scientific, MA, USA) were used to separate and prepare cytoplasmic and nuclear extracts from ESCC cells. Western blotting was performed as described previously [11]. Antibodies against human $\beta$-actin, MAP2K3, p-MAP2K3, EGFR, p-EGFR, STAT1, p-STAT1, STAT3, p-STAT3, caspase 3, cleaved(cl-) caspase 3, GFP, Ki67, Flag, Ub, HA, MDM5, cleaved(cl-) PARP were purchased from Cell Signaling Technology (MA, USA).

\section{Immunohistochemistry (IHC)}

IHC staining was performed using the EnVision System using peroxidase labeling (Dako, Carpinteria, CA) as described previously[11]. IHC staining was examined by two pathologists who were blinded to the clinical outcome. Expression of MAP2K3, STAT3 or indicated protein was analyzed by an individual labeling score considering the proportion of positively stained tumor cells and the intensity of staining. Intensity of stained cells was graded into four levels: 0 : negative staining; 1 : weak staining; 2 : mild staining; and 3: strong staining. The area of staining was evaluated and recorded as a percentage: 0 : no staining; 1 : positive staining in $1-25 \%$ of tumor cells; $2: 26 \%$ to $50 \% ; 3: 51 \%$ to $75 \% ; 4:>76 \%$ of tumor cells. Intensity and fraction of positive cell scores were multiplied, and in this scoring system, low expression was defined as having scores of 0-6, and high expression was defined when the score was 8-12.

\section{Chemicals, plasmids transfection and stable cell lines established}

Cycloheximide (CHX), 5-aza-2'-deoxycytidine (5-aza) were purchased from MCE (NJ, USA). All drugs were dissolved in dimethyl sulfoxide (DMSO; Sigma, St. Louis, MO, USA). The Flag-wild-type (wt) MAP2K3 plasmid, dominant-negative MAP2K3 (S-A), constituted activated MAP2K3(S-E) constructed by replacing Ser-189 and Thr-193 with Ala or Glu residues, Flag-MDM, MDM ${ }^{\text {C464A }}$, GFP-STAT3 wt, STAT3 (Y705A), STAT3 (S727A), MIR19B transcriptional activity reporter plasmids and three mutant type plasmids (mt1: -1462/-1452; mt2: -1295/-1285; mt3: both mutant) were purchased from Vigene (Guangzhou, China). Transfection of plasmids was performed using Lipofectamine 3000 reagent (Invitrogen, NY, USA) according to the manufacturer's instructions.

In addition, the miR-19b-3p mimic or miR-19b-3p inhibitor, and MAP2K3 siRNA were purchased from GenePharma (Jiangsu, China). Transfection of siRNA or miRNA mimics or inhibitors was performed 
using Lipofectamine RNAiMAX (Invitrogen) according to the manufacturer's instructions. Lentivirus encoding a miR-19b-3p mimic or miR-NC was packaged in human embryonic kidney 293T cells and the virus was collected according to the manufacturer's instructions. Stable cell lines were established by infecting ESCC cells with lentivirus, followed by puromycin selection.

\section{Invasion assay}

Cell invasion ability was tested using transwell invasion assay. Briefly, $100 \mu$ l Matrigel (BD, USA) was firstly added onto the bottom of the transwell chamber ( 24 well insert; $8 \mathrm{~mm}$ pore size, Corning Costar Corp), then $1 \times 105$ cells in reduced serum medium (Opti-MEM, Gibco) were placed on the coated membrane in the chamber. Migrated cells on the permeable membrane were fixed and then stained with crystal violet.

\section{Cell proliferation and apoptosis assay}

The viability of ESCC cells was determined with a Cell Counting Kit 8 (CCK8, APExBio, Houston, TX, USA). $5 \times 10^{3}$ of ESCC cells were seeded in each well of a 96 -well plate and allowed to adhere overnight. The change in cell number was determined every 24 hours. Cell density was determined by addition of CCK 8 and measuring OD450 $\mathrm{nm}$ with a microplate reader.

Tumor cells were pretreated or transfected and collected by trypsinization to wash twice with ice-cold PBS. Then then cells were stained using an FITC Annexin/V Apoptosis Detection Kit (BD Pharmingen TM, USA) and quantified by flow cytometry (BD Biosciences, USA).

\section{Colony formation assay}

After transfection or drug treatment, cells were plated in six-well plates, at a density of 500 cells/well, and incubated for 10 days at $37^{\circ} \mathrm{C}$. The cells were then fixed with $4 \%$ buffered formalin for $15 \mathrm{~min}$ and then stained with $1 \%$ crystal violet (Sigma Aldrich, China) for $30 \mathrm{~min}$. The plates were gently washed with PBS and dried before microscopic evaluation. Cell clusters with $>30$ cells were considered as a colony.

\section{Luciferase reporter assay}

Transcriptional activity was measured with a Dual-Luciferase reporter assay system (Promega) according to the manufacturer's instructions. Cells were co-transfected with the dual-luciferase reporter and indicated plasmids or miRNA mimic using Lipofectamine 3000 or RNAiMAX (Invitrogen). Each group was run in triplicate in 48-well plates. The luciferase activity was detected by Dual-Luciferase Reporter Assay System (Promega) after $48 \mathrm{~h}$ of transfection.

\section{Immunocytochemistry}

Cells ( $3 \times 10^{4}$ cells) grown on cover slips were fixed with $4 \%$ paraformaldehyde, permeabilized with $0.1 \%$ Triton $\mathrm{X}-100$, blocked in $3 \% \mathrm{BSA}$, and incubated with primary antibodies overnight at $4^{\circ} \mathrm{C}$. Then, the 
samples were incubated with an Alexa Fluor 488-conjugated anti-mouse IgG fragment (Invitrogen, 1:200) or an Alexa Fluor 555-conjugated anti-rabbit lgG fragment (Invitrogen, 1:200) at room temperature for 30 min in the dark, and were counterstained with 4',6-diamidino-2-phenylindole (DAPI, Invitrogen, 1:300) to stain nuclei. Cells were observed and imaged using a laser-scanning confocal microscope (Leica Microsystems, Germany).

\section{RNA extraction and quantitative RT-PCR}

Using a RNeasy Mini Kit (Qiagen, Valencia, California), total cellular RNA was extracted from cells. Quantitative RT-PCR was performed according to previous published papers using the SYBR system (Takara, China) [18]. The mRNA levels in cell and tissue lysates were normalized against $\beta$-actin. The expression of indicated genes was normalized to an endogenous control by using the 2-DDCt method. Sequences of primers used for qRT-PCR in this study are listed in Supplemental Table 1.

\section{Co-immunoprecipitation (Co-IP)}

Co-IP was performed using special antibodies and protein G-conjugated Dynabeads (Invitrogen) according to the manufacturer's instructions. In brief, cell lysates were incubated with antibodies overnight at $4^{\circ} \mathrm{C}$. The next day, Protein G-conjugated beads were added into the lysate at $4^{\circ} \mathrm{C}$ for $2 \mathrm{~h}$. Then, the beads were washed extensively and boiled in SDS loading buffer. Western blotting was used to detect the immunoprecipitated proteins.

\section{Chromatin immunoprecipitation (ChIP) assays}

ChIP assays were essentially performed as protocol described with slight modifications: $5 \times 10^{6}$ cells were fixed with formaldehyde ( $1 \%$ final volume concentration, Sigma) for $10 \mathrm{~min}$ at room temperature. Fixation was stopped with the addition of $1 / 10$ volume $1.25 \mathrm{M}$ glycine and the samples were incubated for 5 min at room temperature. The sonication step was performed in a qsonica sonicator ( $5 \mathrm{~min}, 20 \mathrm{~s}$ on, $20 \mathrm{~s}$ off), and $200 \mu \mathrm{g}$ of the protein-chromatin complex was used in each immunoprecipitation. Antibody-protein complexes were captured with preblocked dynabeads protein $\mathrm{G}$ (Invitrogen). ChIP DNA was analyzed by qPCR with SYBR Green (Biorad) on an ABI-7500 (Applied Biosystems) using the primers specified in Supplemental Table 1. The antibodies used are as followed: Anti-STAT3 and normal mouse lgG.

\section{RNA sequence}

Total RNA was extracted using Trizol reagent kit (Invitrogen, Carlsbad, USA) according to the manufacturer's protocol. RNA quality was assessed on an Agilent 2100 Bioanalyzer (Agilent Technologies, Palo Alto, CA, USA) and checked using RNase free agarose gel electrophoresis. After total RNA was extracted, eukaryotic mRNA was enriched by Oligo(dT) beads, while prokaryotic mRNA was enriched by removing rRNA by Ribo-Zero ${ }^{\mathrm{TM}}$ Magnetic Kit (Epicentre, Madison, WI, USA). Then the enriched mRNA was fragmented into short fragments using fragmentation buffer and reverse transcripted into cDNA with random primers. Second-strand cDNA were synthesized by DNA polymerase I, RNase H, dNTP 
and buffer. Then the cDNA fragments were purified with QiaQuick PCR extraction kit (Qiagen, Venlo, The Netherlands), end repaired, poly(A) added, and ligated to Illumina sequencing adapters. The ligation products were size selected by agarose gel electrophoresis, PCR amplified, and sequenced using Illumina Novaseq 6000 by Gene Denovo Biotechnology Co. (Guangzhou, China).

\section{Animal studies}

All animal experiments were undertaken in accordance with the NIH Guide for the Care and Use of Laboratory Animals, and with the approval of the institutional animal care and use committee at Sun Yatsen University Cancer Center. For in vivo experiments, indicated treatment and control cells $\left(1 \times 10^{7}\right)$ were injected into the left and right oxter flank of 5-week-old BALB/C nude mice, respectively. Tumor formation was monitored over a 4-week period. Tumor volume was calculated by the formula: tumor volume $=$ $0.5 \times$ length $\times$ width $^{2}$. Tumor volume and body weight were measured every 3 days, and the tumor growth curve was plotted using the tumor volume on the vertical axis and day of measurement following tumor cell implantation on the horizontal axis.

\section{Statistical analysis}

Data are expressed as mean $\pm S D$ of at least three separate experiments. The significance of differences between groups was estimated by Student's t-test or Wilcoxon test, as appropriate. Overall survival rates were calculated by the Kaplan-Meier method with the log-rank test applied for comparison. Survival data were evaluated using univariate and multivariate Cox proportional hazards models using statistical software (SPSS, IBM, USA). A $p$-value of $\leq 0.05$ was considered significant.

\section{Results}

\section{MAP2K3 inhibits cell proliferation and invasion in ESCC both in vitro and in vivo}

To identify the kinase responsible for cell survival, we transduced three ESCC cell lines, KYSE520, KYSE150 and TE1, with a CRISPR knockout (GeCKOv.2) library, targeting the kinome, at a multiplicity of infection $(\mathrm{MOI})<0.3$. We exposed transduced and control tumor cells for 5 weeks in independent screens and observed that the distribution of the sgRNA reads in library transfected samples compared to that of controls was significantly altered in screens (Supplemental Fig. 1A, B). We selected the candidate genes based on the RNAi Gene Enrichment Ranking metric and identified 5 genes, CRKL, MAP2K3, GK5, CERK and WEE2, identified as hits by multiple sgRNAs. These genes were then validated using cell competition assays in the three ESCC lines (Fig. 1A). These selected genes were subjected to loss-of-function analysis in ESCC cells by sgRNA (Supplemental Fig. 1C). Notably, interference of MAP2K3 suppressed cell proliferation compared to the remaining four genes. Therefore, we focused on MAP2K3, an important gene in mitogen-activated protein kinase (MAPK) signaling.

To support the functional role of MAP2K3, we measured cell proliferation, colony formation and invasion ability after MAP2K3 transfection. As shown in Fig. 1B, MAP2K3 transfection increased the expression of 
Ser-189 and Thr-193 phosphorylation (p-MAP2K3) in ESCC cell lines, KYSE150 and KYSE520. Both ESCC cells with MAP2K3 transfection showed a significant decrease in cell proliferation and colony formation detected by CCK8 and colony formation assay, respectively (Fig. 1C, D). MAP2K3 transfection increased apoptosis compared to the negative control cells, as detected by flow cytometry and western blot (Fig. 1E, F). Meanwhile, silencing of MAP2K3 resulted in the opposite behavior in ESCC cells (Fig. 1C-F). To determine the effect of MAP2K3 on ESCC function in vivo, we established a stable MAP2K3-knockout (KO) cell line by using CRISPR/Cas9 in KYSE520 cells. Control or KO cells were then injected into the flanks of nude mice ( $\mathrm{n}=5$ each group). Tumors in the KO group grew faster and heavier than in the controls group (Fig. 1G, H). In addition, IHC was performed to detect the tumor growth in each group by $\mathrm{Ki} 67$ and MAP2K3 staining. Expression of Ki-67 in the MAP2K3-KO group was significantly higher than in the control group (Fig. 1I). Collectively, these in-vitro and in-vivo data demonstrated that loss of MAP2K3 promotes cell proliferation and invasion in ESCC cells.

\section{The function of MAP2K3 in ESCC dependent on its phosphorylation sites}

The MAP2K3 protein kinase is activated by dual phosphorylation on Ser-189 and Thr-193, therefore, we detected whether these phosphorylation sites played a role in the function of MAP2K3 in ESCC. We constructed the dominant-negative MAP2K3(S-A) plasmid and constitutively activated MAP2K3(S-E) by the replacing of Ser-189 and Thr-193 with Ala-189 and Ala-193 or Glu-189 and Glu-193. As shown in Fig. 2A, transfection of MAP2K3(S-A) significantly increased the total MAP2K3 but not p-MAP2K3 expression, as detected by immunofluorescence. Compared to the control group, the MAP2K3(S-E) plasmid transfection inhibited cell growth, colony formation and cell invasion in ESCC (Fig. 2B-D). Furthermore, MAP2K3(S-E) increased the cell apoptosis as detected by western blot and flow cytometry (Fig. 2E-F). However, MAP2K3(S-A) abolished the anti-tumor effect. These results are similar to those observed in the wild-type MAP2K3 transfection, suggesting that Ser-189 and Thr-193 phosphorylation sites affect MAP2K3's function on cell proliferation and invasion in ESCC.

\section{MAP2K3 is down regulated in ESCC and correlates with patient's survival}

To further support the function of MAP2K3 in ESCC, we investigated the expression of MAP2K3 in a cohort of ESCC samples. First, for the GSE20347 $(n=34)$ and the GSE23400 $(n=106)$ cohorts in the kinBase database, the expression of MAP2K3 was significantly lower in ESCC than in paired nontumor tissues (Fig. 3A). Next, we found MAP2K3 was primarily expressed in the cytoplasm of ESCC cells, as detected by immunofluorescence. This result was further supported by examining nuclear and cytoplasmic extracts from in ESCC cell lines (Fig. 3B). Then we identified MAP2K3 expression in six esophageal cell lines, including five ESCC cell lines KYSE150, KYSE520, TE1, KYSE410, and KYSE180, and the NE1 immortalized esophageal epithelial cell line. Compared to NE1, expression of p-MAP2K3 and total MAP2K3 was lower in ESCC cells (Fig. 3C). As shown in Fig. 3D, elevated protein expression of MAP2K3 was observed by IHC in normal epithelial $(n=140)$ when compared to case matched carcinoma in situ (CIS) $(n=12)$ and ESCC tissue $(n=140)$. By western blot, MAP2K3 protein expression was lower in ESCC compared to the case-matched normal epithelial tissues in the majority of ESCC cases (18/24) 
(Fig. 3E). Consistent with the protein expression, the MAP2K3 mRNA expression level were significantly lower in ESCC compared to the normal epithelial cells (Fig. 3F). Furthermore, MAP2K3 expression was significantly correlated with ESCC patient age and tumor differentiation, with well differentiated tumors displaying stronger MAP2K3 expression than poorly differentiated tumors (Table 1). Survival data was available in 70 cases and Kaplan-Meier analysis indicated that ESCC patients with high MAP2K3 expression tended to have a better overall survival, which is consistent with the survival data from TCGA ( $P=0.11$, log-rank test, Fig. $2 \mathrm{G}$ and Supplemental Fig. 2A, B). Moreover, univariate and multivariate Cox regression analyses further confirmed MAP2K3 expression as an independent predictor of survival in ESCC patients (Fig. 2H). 
Table 1

The correlation between MAP2K3 expression and clinical pathology parameters in ESCC

\begin{tabular}{|c|c|c|c|c|c|}
\hline \multirow[t]{2}{*}{ Parameters } & & \multirow{2}{*}{$\begin{array}{l}\text { Case } \\
\mathrm{n}=140\end{array}$} & \multicolumn{2}{|l|}{ Expression } & \multirow[t]{2}{*}{ Result } \\
\hline & & & $\operatorname{High}(n=52)$ & $\operatorname{Low}(n=88)$ & \\
\hline \multirow[t]{2}{*}{ age } & $\leq 57$ & 62 & 14 & 48 & \\
\hline & $>58$ & 78 & 38 & 40 & $P<0.01 *$ \\
\hline \multirow[t]{2}{*}{ Gender } & male & 106 & 44 & 62 & \\
\hline & female & 34 & 8 & 26 & $p=0.264$ \\
\hline \multirow[t]{3}{*}{ Differentiated } & poor & 48 & 28 & 20 & \\
\hline & mid & 86 & 26 & 60 & \\
\hline & well & 6 & 6 & 0 & $p<0.01 *$ \\
\hline \multirow[t]{3}{*}{ Location } & Upper & 16 & 8 & 8 & \\
\hline & Middle & 108 & 38 & 70 & \\
\hline & Lower & 16 & 6 & 10 & $p=0.879$ \\
\hline \multirow[t]{2}{*}{ Tumor size } & $>5 \mathrm{~cm}$ & 46 & 16 & 30 & \\
\hline & $<5 \mathrm{~cm}$ & 94 & 36 & 58 & $p=0.31$ \\
\hline \multirow[t]{3}{*}{ Clinical stage } & II & 74 & 32 & 42 & \\
\hline & III & 62 & 20 & 42 & \\
\hline & IV & 4 & 0 & 4 & $p=0.442$ \\
\hline \multirow[t]{2}{*}{ Adventitia invasion } & $\mathrm{T} 1-\mathrm{T} 2$ & 32 & 14 & 18 & \\
\hline & T3-T4 & 108 & 38 & 70 & $p=1$ \\
\hline \multirow[t]{2}{*}{ Lymph node metastasis } & No & 78 & 38 & 40 & \\
\hline & Yes & 62 & 22 & 40 & $p=0.337$ \\
\hline \multirow[t]{2}{*}{ Distal metastasis } & No & 136 & 52 & 84 & \\
\hline & Yes & 4 & 0 & 4 & $p=0.502$ \\
\hline
\end{tabular}

1. MAP2K3 inhibits the EGFR/STAT3 signaling pathway by promoting STAT3 proteasome degradation

We next explored the mechanisms mediated by MAP2K3 in ESCC. RNA-seq was performed to identify differentially expressed genes between MAP2K3-overexpressing and control cells in three ESCC cell lines, KYSE150, KYSE520 and TE1. There were 693 upregulated genes and 304 downregulated genes and 9 of which were identified in all cell lines after applying a 2-fold screening filter (Supplemental Fig. 3A). Seven 
of the overlapping differentially expressed genes were subjected to validation by qRT-PCR (fold change > 3, Supplemental Fig. 3B). Notably, Gene Ontology (GO) analysis revealed that the type I and II interferon (IFN) signaling pathway was one of the most significantly activated pathways mediated by MAP2K3 in ESCC (Figs. 4A). Then the expression of EGFR, p38 and signal transducer and activator of transcription (STAT)1 and STAT3 were detected in response to MAP2K3 transfection. Overexpression of MAP2K3 increased p38 and STAT1, while decreasing EGFR and STAT3 phosphorylation and total expression. However, knockdown of MAP2K3 also increased both p38 and STAT1 expression in ESCC cells, indicating that EGFR and STAT3 are regulated by MAP2K3 in ESCC (Figs. 4B, C). Since STAT3 is an important transcription factor in tumorigenesis, we further detected the effect of MAP2K3 on STAT3 transcription activity. As shown in Fig. 4D, MAP2K3 decreased STAT3 transcription activity and the mRNA expression of downstream STAT3 genes, such as cyclin D1, survivin and vascular endothelial growth factor (VEGF).

We subsequently further explored how MAP2K3 mediates expression of STAT3 in ESCC. First, we found that MAP2K3 and STAT3 were co-localized in the cytoplasm as detected by immunofluorescence confocal microscopy (Fig. 4E). Furthermore, bands specific to MAP2K3 or STAT3 were obtained by coimmunoprecipitation (co-IP) assay and subjected to western blot analysis for protein identification. STAT3-MAP2K3 interaction was higher in the MAP2K3-overexpressing group than in the control group (Fig. 4F). Previous research demonstrated that STAT3 is downregulated in a ubiquitin proteasome degradation-dependent manner. Treatment of ESCC cells with MG132 resulted in increased expression of the STAT3 protein (Fig. 4G). Then we detected whether MAP2K3 affects ubiquitination and degradation of the STAT3 protein. The half-life of STAT3 was significantly increased in MAP2K3 knockdown cells as detected by cycloheximide chase assay (Fig. 4H). Ubiquitination assay suggested that STAT3 protein ubiquitination was increased in MAP2K3 overexpressing cells as compared to the control cells (Fig. 4I). Tyrosine 705 (Y705) and serine 727 (S727) phosphorylation sites on STAT3 play an important role in STAT3 degradation. We found that MAP2K3 binds with both wildtype and mutant STAT3 but did not increased the polyubiquitination of STAT3 S727A (Supplemental Fig. 3C and Fig. 4J). MAP2K3 increased ubiquitination of STAT3 on the K48-linked ubiquitin chain, but not the K63-linked ubiquitin chain (Fig. 4K). These findings suggest that the degradation of STAT3 mediated by MAP2K3 is dependent on the STAT3 Y705 phosphorylation site.

\section{MAP2K3 interacts with the E3 ligase MDM2 to promote STAT3 degradation}

We next investigated which E3 ubiquitin ligase mediates the protein degradation of STAT3 induced by MAP2K3. Mouse double minute 2 (MDM2) was predicted with the highest confidence as a primary E3 ligase for STAT3 in the UbiBrowser database (Supplementary Fig. 4A). We then confirmed a stronger interaction between endogenous STAT3 and MDM2 after MAP2K3 transfection (Fig. 5A), suggesting that MDM2 associates with STAT3 in ESCC cells. Moreover, the tyrosine 705 (Y705) and serine 727 (S727) phosphorylation site mutations of STAT3 abolished the observed interactions with MDM2 (Fig. 5B). The SH2 domain deletion of STAT3 also abolished the interactions (Fig. 5C). Taken together, our data suggest that MDM2 binds to STAT3 via the STAT3 SH2 domain. 
To test whether MDM2 affects cellular levels of STAT3, we overexpressed wild type (wt) MDM2 in KYSE150 cells and found that the endogenous protein level of STAT3 was sharply reduced. However, ectopic expression of MDM2 ${ }^{\mathrm{C} 464 \mathrm{~A}}$, which lacks ubiquitin ligase activity, did not affect levels of STAT3, indicating that the E3 catalytic activity of MDM2 is required for STAT3 protein destabilization (Fig. 5D). Consistently, the half-life of STAT3 was significantly reduced in MDM2 overexpressing cells (Fig. 5E) but not in MDM2 ${ }^{\mathrm{C} 464 \mathrm{~A}}$ overexpressing cells (Supplementary Fig. 4B) as detected by cycloheximide chase assay. These results suggest that MDM2 is the E3 ligase that destabilizes STAT3 in ESCC cells. To investigate whether endogenous MDM2 contributes to MAP2K3-induced protein degradation of STAT3, we transfected KYSE150 cells with MDM2 siRNA. Depletion of MDM2 resulted in a slight increase in the amount of STAT3, and this effect of MDM2 was more substantial after MAP2K3 treatment (Fig. 5F). Consistently, knockdown of MDM2 extended the half-life of STAT3, and the effect of MDM2 was more significant after MAP2K3 treatment (Fig. 5G). Next, we investigated whether MDM2 promotes ubiquitination of STAT3. As shown in the ubiquitination assays, overexpression of MDM2 significantly increased the ubiquitination of STAT3, but MDM2 ${ }^{\mathrm{C} 464 \mathrm{~A}}$ did not. Consistent with these observations, we found that knockdown of MDM2 markedly reduced the ubiquitination of STAT3 in KYSE150 cells (Fig. $5 \mathrm{H}$ ). By analyzing the lysine residues within the $\mathrm{SH} 2$ domain for possible ubiquitination sites, we mutated a predicted $\mathrm{K} 631$ lysine residues to Ala. We found that STAT3 ubiquitination was substantially abolished in the STAT3 K631A mutant (Fig. 5I). Taken together, these results demonstrate that MAP2K3 interacts with the E3 ligase MDM2 to promote STAT3 degradation.

\section{STAT3 is an essential factor in MAP2K3-mediated tumorigenesis in ESCC}

To investigate whether STAT3 is involved in MAP2K3-mediated tumorigenesis in ESCC, we transfected ESCC cells with a plasmid encoding STAT3 and treated the cells. Compared to the control cells, STAT3 overexpression significantly promoted KYSE150 and KYSE520 cell proliferation and colony formation (Supplemental Fig. 5A, B), and western blot analysis indicated that STAT3 overexpression significantly diminished the expression of apoptotic biomarkers (Supplemental Fig. 5C). Conversely, STAT3 knockdown significantly attenuated KYSE150 and KYSE520 cell proliferation and clonogenicity. Collectively, these results suggest that STAT3 functions as oncogene in ESCC. We also aimed to detect whether MAP2K3 mediates ESCC cell proliferation and invasion through suppressing STAT3 by performing rescue experiments involving overexpressing STAT3 in MAP2K3-transfected cells and knockdown of STAT3 in si-MAP2K3 transfected cells. Transfection efficiency is shown in Fig. 6A. As shown in Fig. 6B-D and Supplemental Fig. 5D, colony formation, cell invasion and proliferation experiments suggested that overexpression of STAT3 abolished MAP2K3-inhibited cell proliferation and invasion. Conversely, knockdown of STAT3 by siRNA partially reversed the cell proliferation and invasion ability induced by loss of MAP2K3 (Fig. 6E).

To determine whether a clinical correlation exists between MAP2K3 and STAT3 expression, we analyzed their expression in 140 ESCC tissues by IHC staining (Fig. 6F). STAT3 expression was 1.53-fold higher in ESCC compared to case-matched normal epithelial tissues and was negatively correlated with MAP2K3 expression. Furthermore, the in vivo experiment supporting our in vitro results, showed that tumor size 
and weight were significantly higher in MAP2K3-KO tumors compared to controls, while STAT3 knockdown tumors had the smallest tumors (Fig. 6G, H). The immunohistochemistry of Ki-67 and STAT3 was performed and revealed that STAT3 and Ki-67 expression was significantly higher in the MAP2K3-KO group, while knockdown of STAT3 abolished this phenomenon, indicating that STAT3 knockdown diminished tumor activity induced by MAP2K3-KO (Fig. 6I and Supplemental Fig. 5E). Base on the above results, we concluded that STAT3 is an essential factor in MAP2K3-mediated tumorigenesis in ESCC

\section{Mir-19b-3p Targets Map2k3 To Promote Escc Tumorigenesis}

To uncover the mechanism by which MAP2K3 is down regulated in ESCC, we performed several experiments. However, we found that low expression of MAP2K3 in ESCC is caused by neither proteasome degradation nor promoter methylation (Supplemental Fig. 6A). MiRNAs are involved at the posttranscriptional level and bind to the 3' UTRs of their target mRNAs to suppress gene expression. Thus, using predictions of Targetscan, DIANA-TarBase and miRTarBase databases, we identified 3 miRNAs (miR-21-5p, miR-19a-3p and miR-19b-3p) in common that could serve as potential upstream regulators of MAP2K3 (Fig. 7A). PCR analysis revealed that all three miRNAs mimics decreased MAP2K3 mRNA expression, with miR-19b-3p being the most significant. Using 3' UTR luciferase reporter assays, we found that a miR-19b-3p mimic inhibited luciferase activity of a MAP2K3-dependent reporter gene, while mutant vectors had no influence, and a miR-19b-3p inhibitor induced the opposite effect (Supplemental Fig. 6B). A miR-19b-3p mimic suppressed MAP2K3 RNA and protein expression, while a miR-19b-3p inhibitor increased MAP2K3 expression (Fig. 7A and Supplemental Fig. 6C). These results indicate that miR-19-3p represses expression of MAP2K3 through binding the $3^{\prime} U T R$ of its mRNA.

Then, we determined whether miR-19b-3p plays a role in ESCC tumorigenesis. First, we transfected the miR-19-3p mimic into ESCC cells that exhibit decreased apoptosis and increased cell survival, colony formation and cell invasion, compared to control cells (Fig. 7B-E). ESCC cells transfected with the miR$19 b-3 p$ inhibitor exhibited the opposite results. These data support our in vitro findings, indicating that miR-19-3p promotes ESCC tumorigenesis. Then, we analyzed the correlation between miR-19b-3p and MAP2K3 expression in ESCC samples. Expression of miR-19b-3p was higher in ESCC compared to normal esophageal epithelium as detected by qRT-PCR (Fig. 7F), and there was an inverse correlation between MAP2K3 and miR-19b-3p expression in ESCC (Supplemental Fig. 6D). To determine whether loss of MAP2K3 is responsible for miR-19b-3p-mediated cell proliferation and invasion, we performed rescue experiments. ESCC cells transfected with a miR-19b-3p mimic displayed decreased expression of MAP2K3, which was restored by the MAP2K3 plasmid in recipient cells. Concurrent overexpression of MAP2K3 abolished miR-19b-3p-mediated cell survival, as detected by colony formation (Figs. 7G). Conversely, knockdown of MAP2K3 abrogated the ability of the miR-19b-3p inhibitor to increase apoptosis. Taken together, these results indicate that miR-19b-3p mediates ESCC tumorigenesis by suppressing MAP2K3. 


\section{STAT3 binds to the MIR19B promoter to increase miR-19-3p expression}

STAT3 is an important transcription factor in tumor progression. Inspection of the MIR19B genomic region revealed a conserved STAT3-binding according to the JASPAR website (http://jaspar.genereg.net/analysis) and two STAT3 binding sites were identified (-1462/-1452 and 1295/-1285 sites). Thus, we examined whether STAT3 directly binds to the MIR19B promoter to increase its expression. First, we analyzed the expression of miR-19b-3p expression in response to STAT3 transfection or knockdown. As shown in Fig. 8A, STAT3 increased, while si-STAT3 decreased expression of miR-19b-3p in both KYSE150 and KYSE520 cells. We further found that STAT3 overexpression increased expressions of miR-19b-3p in a dose-dependent manner (Fig. 8B). To determine whether STAT3 binds to the MIR19B promoter region, specific primers were designed and synthesized for two potential binding sites in MIR19B, and the binding of STAT3 to these putative sites was demonstrated by ChIP analysis. After transfection of KYSE150 and KYSE520 cells with STAT3, the STAT3 occupancy at the MIR19B promoter significantly increased, as shown by ChIP (Fig. 8C).

There are two consensus STAT3-binding sites located within the promoter region of MIR19B. To determine whether these two STAT3 sites contribute to STAT3-mediated p300 activity, we constructed four reporter plasmids, wide type (wt), mutant (mt)1, mt2, and mt3, with - 1462-STAT3 site, -1295-STAT3 site and both sites mutated, respectively, as illustrated in Fig. 8D. Each of these reporter plasmids was cotransfected with STAT3 or control into 293 T cells, and relative luciferase activity was assessed. Compared to the wild type, both the - 1462-site mutation (mt1) and the - 1295-site mutation ( $\mathrm{mt} 2)$ reduced the synergistic activity of STAT3 on promoter activation. For the double-mutation (mt3), the synergistic activity of STAT3 was decreased far more significantly. To further examine MAP2K3's effect on miR-19b-3p, we performed rescue experiments and found that expression and transcription activity of miR-19b-3p was increased in STAT3 overexpressed cells, while MAP2K3 reduced it. In contrast, STAT3 knockdown decreased expression and transcriptional activity of miR-19b-3p, and overexpression of MAP2K3 restored it (Fig. 8E, F).

\section{Discussion}

In this study, we show that the miR-19b-3p/MAP2K3/STAT3 feedback loop regulates ESCC tumorigenesis. MAP2K3 has lower expression in ESCC than in case-matched normal esophageal epithelium. MAP2K3 promotes apoptosis and inhibits cell growth in ESCC cells and functionally exerts its effects through the downregulation of the EGFR/STAT3 pathway by promoting STAT3 proteasome degradation. In addition, miR-19b-3p mediates cell proliferation and invasion by suppressing STAT3. Moreover, STAT3 binds to the MIR19 promoter region and increases expression of miR-19b-3p in ESCC cells (Fig. 8G).

MAP2K3 is a key mediator of the MAPK signaling pathways and is involved in several biological processes. The role of MAP2K3 in cancer progression is contradictory. MAP2K3 has been shown to enhance tumor progression, and the loss of MAP2K3 results in inhibition of cellular proliferation and 
increased response of tumor cells to chemotherapeutic drugs in vivo $[5,12,13]$. However, recent research has demonstrated that the loss of MAP2K3 copy number occurs in NSCLC and that MAP2K3 inhibits cell proliferation and promotes cellular senescence in hepatocellular carcinoma, breast cancer and melanoma $[7,14-17]$. In this study, we demonstrated that MAP2K3 is downregulated in ESCC compared to casematched normal tissues. We also revealed that ESCC patients with higher MAP2K3 expression have better prognosis. Previous findings have suggested an important role for MAP2K3 in tumor invasion and progression, such as in colon cancer. However, in our study, we found that MAP2K3 inhibits cell proliferation and colony formation, indicating that MAP2K3 plays a tumor suppressor role in ESCC. Loss of MAP2K3 enhances cell proliferation and invasion ability in ESCC.

Furthermore, we demonstrated that MAP2K3 suppresses STAT3 expression in ESCC. STAT3 has been a protein target due to its roles in the progression of cancer development, cancer stemness, chemoresistance and radioresistance [9]. STAT3 is constitutively activated in many cancer types, and such hyperactivation is associated with a poor clinical prognosis in ESCC, breast cancer and lung cancer [18]. Previous research demonstrated that STAT3 overexpression contributes to drug resistance in cancer treatment, such as in targeted therapy, chemotherapy and immunotherapy, but its regulation in tumors has not been clearly demonstrated. In this study, we explored the mechanism underlying MAP2K3mediated downregulation of STAT3, performing the protein stability and ubiquitination assays to show that MAP2K3 may directly bind to STAT3 and promote its proteasomal degradation. Furthermore, we revealed that the E3 ligase MDM2 mediates in this process. MDM2 is known to induce ubiquitination of several target proteins, with the most well-known target p53 [19]. MDM2 upregulation is associated with cancer development by repressing p53 [20]. In this paper, we found that MDM2 interacted with STAT3 to promote STAT3 ubiquitination and this progress was enhanced by MAP2K3 transfection.

In the end, we attempted to identify the mechanisms underlying the downregulation of MAP2K3 in ESCC. MiRNAs regulate gene expression by binding the $3^{\prime} U T R$ of target transcripts in a complete or incomplete complementary binding manner. According to our predicted results, MAP2K3 might be a target gene of miR-19b-3p, a key oncogenic component of the polycistronic miR-17 92 cluster[21]. Then, we identified miR-19b-3p as an upstream regulator of MAP2K3 by luciferase reporter assay. In cancer, miR-19b-3p promotes cell proliferation, invasion, and metastasis in clear cell renal cell carcinoma, lung, colorectal and breast cancer[22-24]. High expression of miR-19b-3p was found in colon cancer and significantly associated with poor patient survival[25]. The role of miR-19b-3p is not without controversy. The miR-19b expression level is significantly down-regulated in breast cancer and may function as a tumor suppressor[26]. However, the expression and function of miR-19b-3p in ESCC development remains unclear. In this study, we reported that miR-19b-3p can promote cell proliferation and suppress apoptosis in ESCC by targeting MAP2K3. and the rescue experiments convince us that the function of miR-19b-3p is partly dependent on suppressing MAP2K3. Furthermore, we identified STAT3 as the transcription driver of miR-19b-3p. We firstly validated that STAT3 upregulates the expression of miR-19b-3p and binds with the promoter region of MIR19B to increase its transcription. Thus, miR-19b-3p, MAP2K3 and STAT3 formed a positively feedback loop to contribute to ESCC progression. 


\section{Conclusion}

In summary, we showed that MAP2K3 was downregulated in ESCC and correlated with clinically poor survival. MAP2K3 inhibited cell proliferation and invasion via the EGFR/STAT3 signaling pathway in ESCC cells. MAP2K3 suppressed STAT3 expression and activation by promoting STAT3 ubiquitin proteasome degradation. Furthermore, we found that miR-19b-3p suppressed MAP2K3 expression and could be transcriptional activated by STAT3 in ESCC cells. Our study is the first to demonstrate that miR19b-3p/MAP2K3/STAT3 feedback loop regulates ESCC tumorigenesis. Therefore, MAP2K3 may serve as a predictor and potential therapeutic target for ESCC.

\section{Abbreviations}

\section{5-aza}

5-aza-2'-deoxycytidine; CCK-8:cell counting kit-8; CHX:cycloheximide; Cl:confidence interval; CIS:carcinoma in situ; CRISPR/Cas9:clustered regularly interspaced short palindromic repeats/associated protein 9; DAPI:4',6-diamidino-2-phenylindole; DMEM:dulbecco' s modified eagle' s medium; EGFR:epidermal growth factor receptor; ESCC:esophageal squamous cell carcinoma; FBS:fetal bovine serum; GO:gene ontology; HR:hazard ratio; IHC:immunohistochemical; IFN:interferon; IP:immunoprecipitation; KO:knockout; MAP2K3:mitogen-activated protein kinase kinase 3; MAPK:mitogen-activated protein kinase; MDM2:Mouse Double Minute 2; miRNAs:microRNAs; NC:negative control cells; NSCLC:non-small-cell lung cancer; qRT-PCR:quantitative reverse transcription polymerase chain reaction; STAT3:Signal transducers and activators of transcription 3; UTRs:3' untranslated regions; VEGF:vascular endothelial growth factor.

\section{Declarations}

\section{Acknowledgements}

We thank Xia Yang (Sun Yat-sen University Cancer Center) for kindly help with plasmids.

\section{Funding}

This work was supported by Natural Science Foundation of Guangdong (2018A030310219), Guangdong Medical Science and Technology Research Fund (A2018518) and the China Postdoctoral Foundation (2019M653195).

\section{Availability of data and materials}

The datasets used for the current study are available from the corresponding author on reasonable request. The authenticity of this article has been validated by uploading the key raw data onto the Research Data Deposit public platform (http://www.researchdata.org.cn/), with the approval RDD number as RDDB2020000866. 


\section{Authors' contributions}

All the authors have precipitated in the conception and design of the study. YZ, ZYL, WQL, YLC, XY have obtained and analyzed the data. YZ, WQL, YLC organized the data and drafted the manuscript. YZ revised the manuscript. All the authors have read and approved the final version of the manuscript.

\section{Ethics approval and consent to participate}

All authors approved and directly participated in the planning, execution and/or analysis of the data presented here. All protocols were approved by the Ethics Committee of Shantou University Medical College and Sun Yat-sen University Cancer Center, and informed consent was obtained from all patients before surgery. All in vivo protocols were approved by the Institutional Animal Care and Use Committee of Shantou University Medical College and Sun Yat-sen University Cancer Center.

\section{Consent for publication}

The content of this manuscript has not been previously published and is not under consideration for publication elsewhere.

\section{Competing interests}

The authors declare no conflicts of interest.

\section{References}

1. Lin DC, Hao JJ, Nagata Y, Xu L, Shang L, Meng X, Sato Y, Okuno Y, Varela AM, Ding LW, et al. Genomic and molecular characterization of esophageal squamous cell carcinoma. Nat Genet. 2014;46(5):467-73.

2. Paez JG, Janne PA, Lee JC, Tracy S, Greulich H, Gabriel S, Herman P, Kaye FJ, Lindeman N, Boggon TJ, et al. EGFR mutations in lung cancer: correlation with clinical response to gefitinib therapy. Science. 2004;304(5676):1497-500.

3. Schadendorf D, van Akkooi ACJ, Berking C, Griewank KG, Gutzmer R, Hauschild A, Stang A, Roesch A, Ugurel S. Melanoma. Lancet. 2018;392(10151):971-84.

4. Bossi G. MKK3 as oncotarget. Aging. 2016;8(1):1-2.

5. Baldari S, Ubertini V, Garufi A, D'Orazi G, Bossi G. Targeting MKK3 as a novel anticancer strategy: molecular mechanisms and therapeutical implications. Cell death disease. 2015;6:e1621.

6. Xie X, Liu K, Liu F, Chen H, Wang X, Zu X, Ma X, Wang T, Wu Q, Zheng Y, et al. Gossypetin is a novel MKK3 and MKK6 inhibitor that suppresses esophageal cancer growth in vitro and in vivo. Cancer letters. 2019;442:126-36.

7. Jia M, Souchelnytskyi N, Hellman U, O'Hare M, Jat PS, Souchelnytskyi S. Proteome profiling of immortalization-to-senescence transition of human breast epithelial cells identified MAP2K3 as a 
senescence-promoting protein which is downregulated in human breast cancer. Proteomics Clinical applications. 2010;4(10-11):816-28.

8. Chai EZ, Shanmugam MK, Arfuso F, Dharmarajan A, Wang C, Kumar AP, Samy RP, Lim LH, Wang L, Goh BC, et al. Targeting transcription factor STAT3 for cancer prevention and therapy. Pharmacol Ther. 2016;162:86-97.

9. Furtek SL, Backos DS, Matheson CJ, Reigan P. Strategies and Approaches of Targeting STAT3 for Cancer Treatment. ACS chemical biology. 2016;11(2):308-18.

10. Banerjee $\mathrm{K}$, Resat H. Constitutive activation of STAT3 in breast cancer cells: A review. International journal of cancer. 2016;138(11):2570-8.

11. Zhang Y, Chen Y, Yun H, Liu Z, Su M, Lai R. STAT1 beta enhances STAT1 function by protecting STAT1alpha from degradation in esophageal squamous cell carcinoma. Cell death disease. 2017;8(10):e3077.

12. Sun Y, Zhang D, Guo X, Li W, Li C, Luo J, Zhou M, Xue L. MKK3 modulates JNK-dependent cell migration and invasion. Cell death disease. 2019;10(3):149.

13. Stramucci L, Pranteda A, Stravato A, Amoreo CA, Pennetti A, Diodoro MG, Bartolazzi A, Milella M, Bossi G. MKK3 sustains cell proliferation and survival through p38DELTA MAPK activation in colorectal cancer. Cell death disease. 2019;10(11):842.

14. MacNeil AJ, Jiao SC, McEachern LA, Yang YJ, Dennis A, Yu H, Xu Z, Marshall JS, Lin TJ. MAPK kinase 3 is a tumor suppressor with reduced copy number in breast cancer. Cancer research. 2014;74(1):162-72.

15. Owusu-Ansah KG, Song G, Chen R, Edoo MIA, Li J, Chen B, Wu J, Zhou L, Xie H, Jiang D, et al. COL6A1 promotes metastasis and predicts poor prognosis in patients with pancreatic cancer. Int $\mathrm{J}$ Oncol. 2019;55(2):391-404.

16. Wang L, Chen C, Feng S, Lei P, Tian J. Mitogen-activated protein kinase kinase 3 induces cell cycle arrest via p38 activation mediated Bmi-1 downregulation in hepatocellular carcinoma. Mol Med Rep. 2016;13(1):243-8.

17. Samulin Erdem J, Skaug V, Haugen A, Zienolddiny S. Loss of MKK3 and MK2 Copy Numbers in NonSmall Cell Lung Cancer. J Cancer. 2016;7(5):512-5.

18. Liu Y, Wang X, Zeng S, Zhang X, Zhao J, Zhang X, Chen X, Yang W, Yang Y, Dong Z, et al. The natural polyphenol curcumin induces apoptosis by suppressing STAT3 signaling in esophageal squamous cell carcinoma. Journal of experimental clinical cancer research: CR. 2018;37(1):303.

19. Jung JH, Bae S, Lee JY, Woo SR, Cha HJ, Yoon Y, Suh KS, Lee SJ, Park IC, Jin YW, et al: E3 ubiquitin ligase Hades negatively regulates the exonuclear function of p53. Cell death and differentiation 2011, 18(12):1865-1875.

20. Carroll PE, Okuda M, Horn HF, Biddinger P, Stambrook PJ, Gleich LL, Li YQ, Tarapore P, Fukasawa K. Centrosome hyperamplification in human cancer: chromosome instability induced by p53 mutation and/or Mdm2 overexpression. Oncogene. 1999;18(11):1935-44. 
21. Quattrochi B, Gulvady A, Driscoll DR, Sano M, Klimstra DS, Turner CE, Lewis BC. MicroRNAs of the mir-17 92 cluster regulate multiple aspects of pancreatic tumor development and progression. Oncotarget. 2017;8(22):35902-18.

22. Li X, Xie W, Xie C, Huang C, Zhu J, Liang Z, Deng F, Zhu M, Zhu W, Wu R, et al. Curcumin modulates miR-19/PTEN/AKT/p53 axis to suppress bisphenol A-induced MCF-7 breast cancer cell proliferation. Phytother Res. 2014;28(10):1553-60.

23. Cellura D, Pickard K, Quaratino S, Parker H, Strefford JC, Thomas GJ, Mitter R, Mirnezami AH, Peake NJ. miR-19-Mediated Inhibition of Transglutaminase-2 Leads to Enhanced Invasion and Metastasis in Colorectal Cancer. Molecular cancer research: MCR. 2015;13(7):1095-105.

24. Zhou J, Zhang X, Shi H, Fan C. MiR-19 regulates breast cancer cell aggressiveness by targeting profilin 1. FEBS Lett. 2017;591(11):1623.

25. Jiang T, Ye L, Han Z, Liu Y, Yang Y, Peng Z, Fan J. miR-19b-3p promotes colon cancer proliferation and oxaliplatin-based chemoresistance by targeting SMAD4: validation by bioinformatics and experimental analyses. Journal of experimental clinical cancer research: CR. 2017;36(1):131.

26. Maleki E, Ghaedi K, Shahanipoor K, Karimi Kurdistani Z: Down-regulation of microRNA-19b in hormone receptor-positive/HER2-negative breast cancer. APMIS: acta pathologica, microbiologica, et immunologica Scandinavica 2018, 126(4):303-308.

\section{Figures}


Fig 1

A
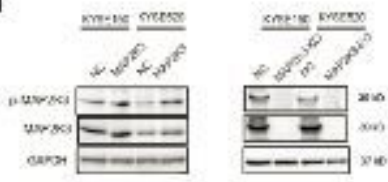

B
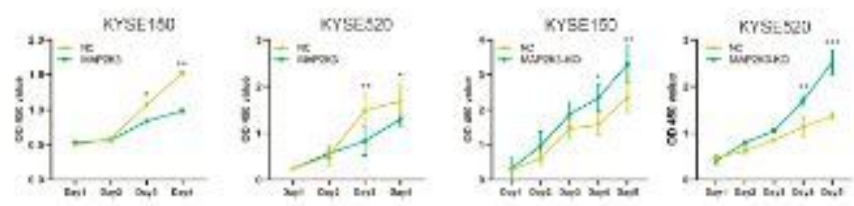

$\mathrm{c}$
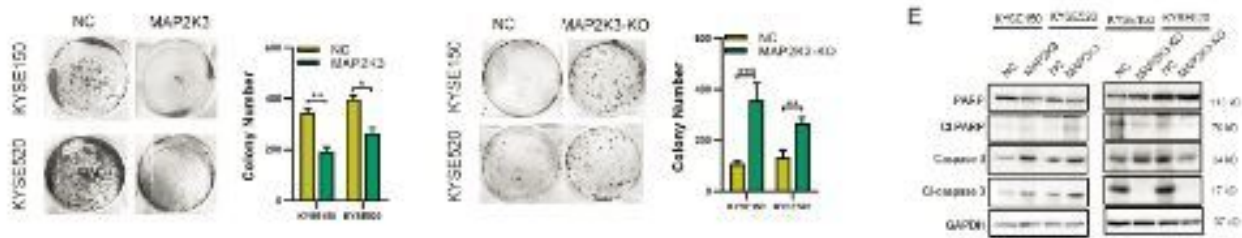

D
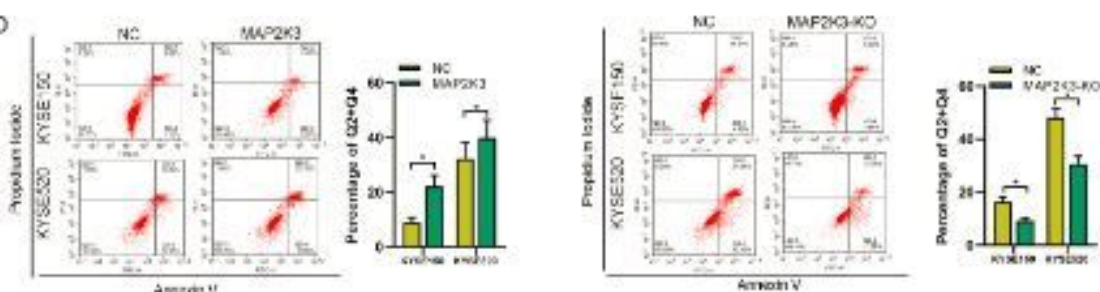

G
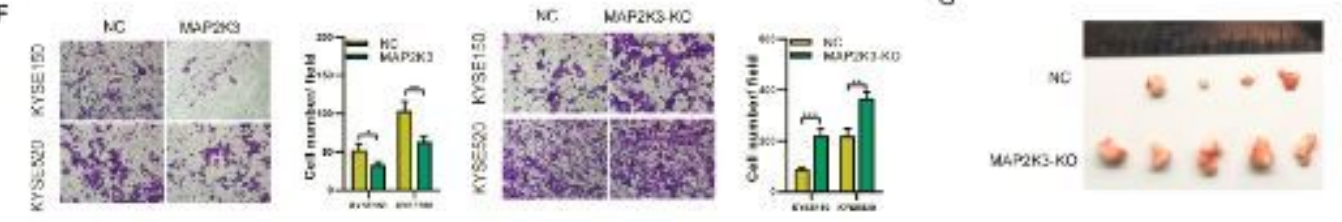

H
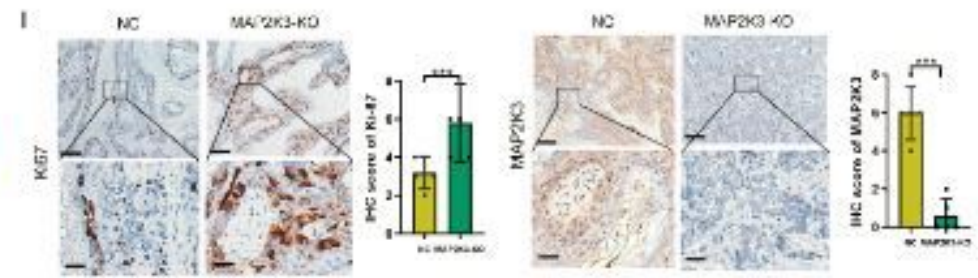

\section{Figure 1}

MAP2K3 inhibited cell proliferation and invasion in ESCC in vitro and in vivo. A. Expression of p-MAP2K3 and MAP2K3 was detected by western blot in KYSE150 and KYSE520 cells after MAP2K3 transfection and treatment. B. Cell growth was detected by CCK8 after MAP2K3 transfection or knockout in KYSE150 and KYSE520 cells. C. Colony formation assay was performed after MAP2K3 transfection or knockout in KYSE150 and KYSE520 cells treated. D. Flow cytometry analysis of cell apoptosis caused by MAP2K3 transfection or knockout in KYSE150 and KYSE520 cells treated. E. Western blot assay to detect apoptosis biomarkers, cleaved (cl-) PARP and caspase 3, in ESCC cells transfected with MAP2K3 or control vector. F. The cell invasion ability was detected by transwell assay. G. Six weeks after KYSE520 MAP2K3-KO and control cells were inoculated into the armpits of nude mice ( $\mathrm{n}=6$ each group). Tumor volume and mouse weight were measured after injection of the indicated ESCC cells. $\mathrm{H}$. The tumor weight and size was measured in the indicated time after injection. I. The representative photographs of immunohistochemistry staining of MAP2K3 and Ki67 in tissues from control and MAP2K3-KO groups of mice (Scale bar: $400 \square \mathrm{m}, 50 \square \mathrm{m}$, respectively). Error bars represent the SD from at least three independent biological replicates. $\left({ }^{\star} p<0.05 ; * \star p<0.01 ; * \star \star p<0.001\right)$ 


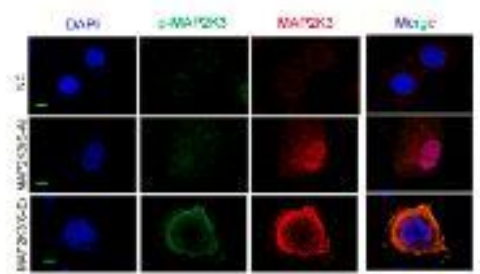

B
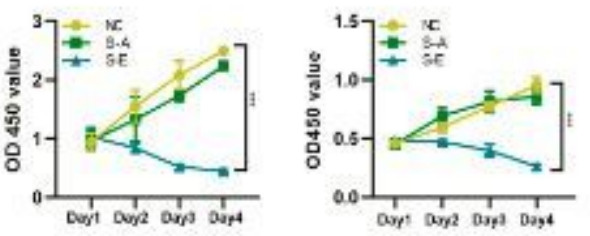

c

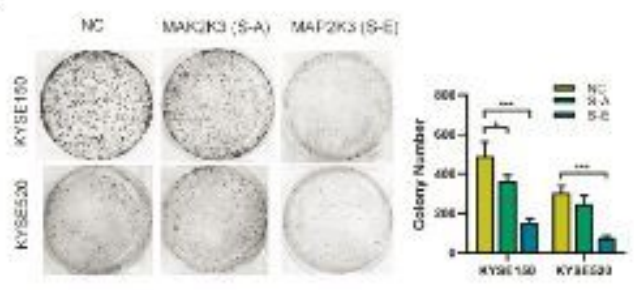

D

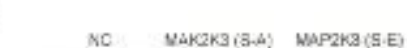

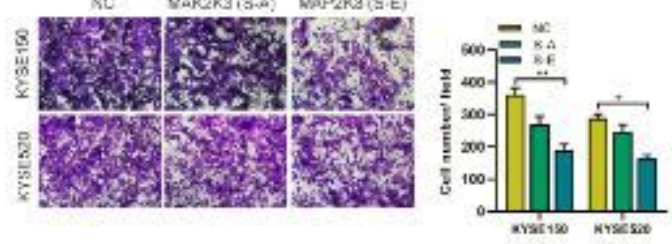

E
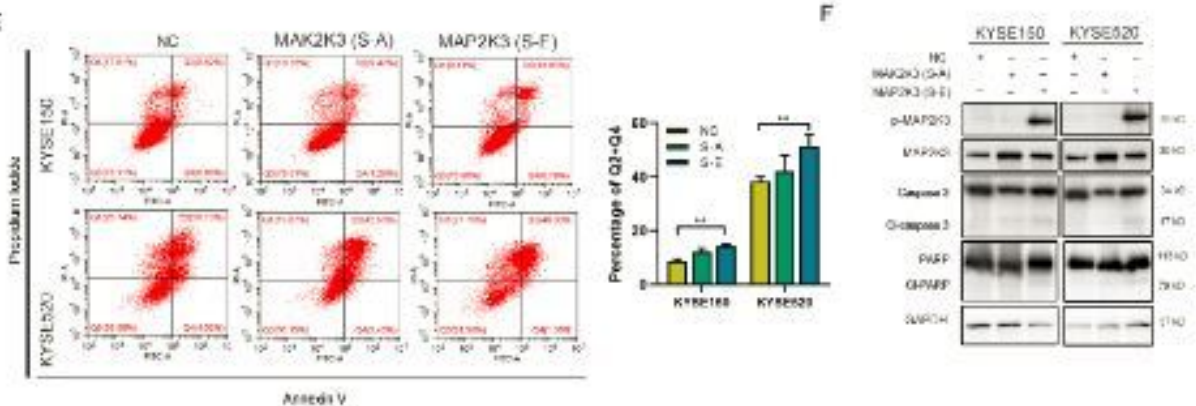

Figure 2

The function of MAP2K3 in ESCC dependent on its phosphorylation sites. A. Expression of p-MAP2K3 and total MAP2K3 was detected by immunofluorescence after wild-type MAP2K3(S-E) and MAP2K3(S-A) mutant plasmids transfection. B. Cell growth was detected by CCK8 assay after MAP2K3(S-E), MAP2K3(S-A) or control plasmids transfection. C. Colony formation after transfection with MAP2K3(S-E), MAP2K3(S-A) or control plasmids. D. Protein expression after transfection with MAP2K3(S-E), MAP2K3(SA) or control plasmids. E. Apoptosis was detected by Annexin V staining after MAP2K3(S-E), MAP2K3(SA) or control transfection. F. Western blot assay to detect apoptosis biomarkers, cleaved (cl-) PARP and caspase 3, in ESCC cells transfected with MAP2K3(S-E), MAP2K3(S-A) or control plasmids. Error bars represent the SD from at least three independent biological replicates. $\left({ }^{\star} p<0.05 ;{ }^{*} p<0.01 ;{ }^{* \star *} p<0.001\right)$. 


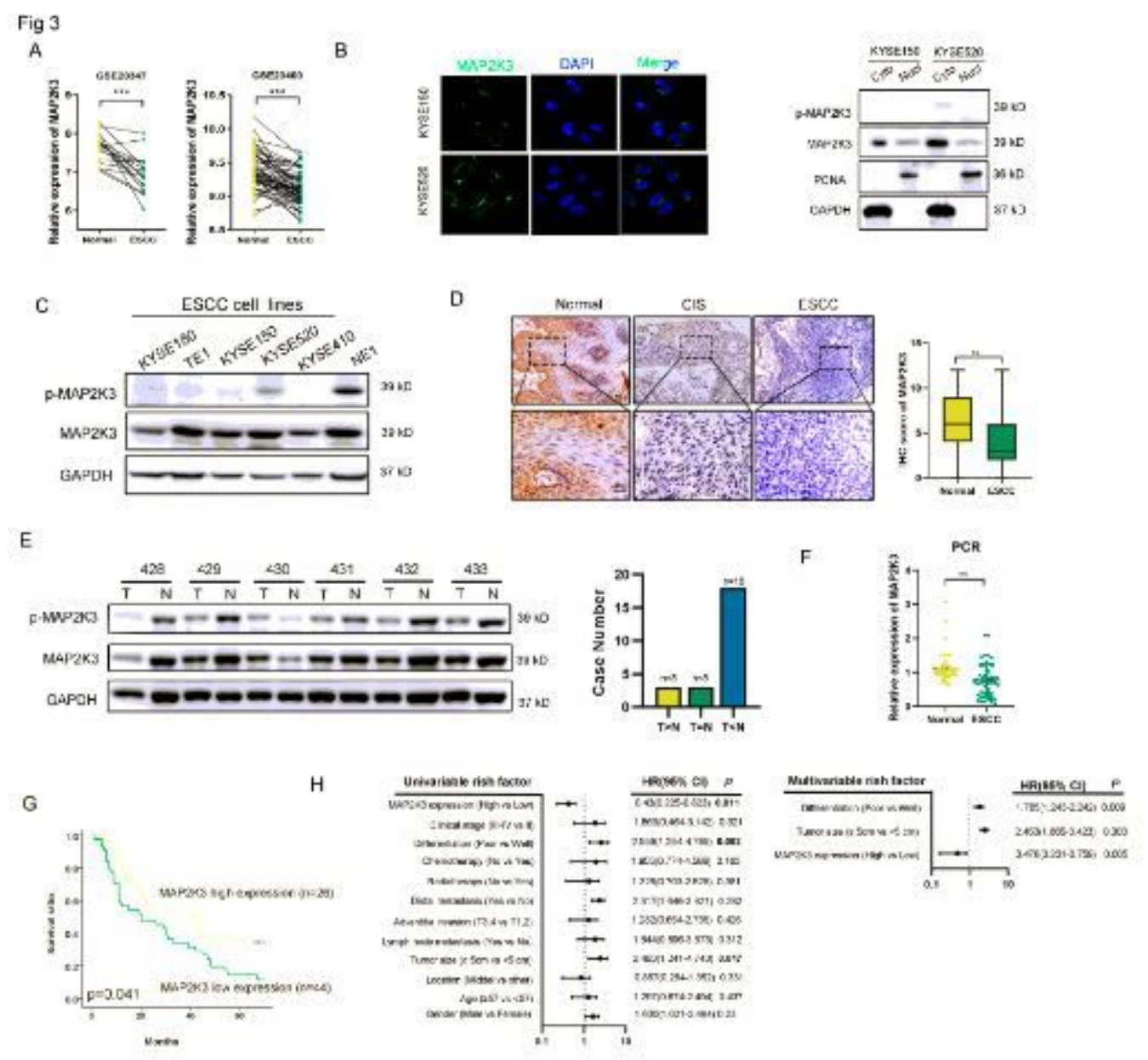

Figure 3

Expression of MAP2K3 in ESCC tissues and its clinical parameters. A. Expression of MAP2K3 in ESCC and case-matched normal epithelium was explored in the GSE database (GSE20347 and GSE23400). B. MAP2K3 expression was detected in KYSE150 and KYSE520 by immunofluorescence and nuclear/cytoplasmic assay. C. Western blot for p-MAP2K3 and MAP2K3 in ESCC cell lines (KYSE180, TE1, KYSE150, KYSE520 and KYSE410) and an immortalized esophageal cell line (NE1). D. Representative IHC detection of MAP2K3 in ESCC, carcinoma in situ (CIS) and normal epithelial tissues( $n=140)$. Immunostaining of MAP2K3 in ESCC and normal groups was scored (Scale bar: $100 \square \mathrm{m}, 20 \mathrm{Im})$. E. Western blot for protein expression of MAP2K3 in ESCC and case matched normal tissues $(n=24)$. F. MAP2K3 was detected in 34 pairs of ESCC and case-matched normal esophageal epithelial tissues by qRT-PCR. G. Kaplan-Meier analysis showed that ESCC patients with high levels of MAP2K3 expression had longer survival times compared with low MAP2K3-expressing patients ( $P=0.041$, log-rank test). $\mathrm{H}$. Forest plot showing the association between MAP2K3 expression and ESCC survival using univariate and multivariate analyses (HR, hazard ratio; $\mathrm{Cl}$, confidence interval). Error bars represent the SD from at least three independent biological replicates. $\left({ }^{\star} p<0.05 ; * \star p<0.01 ; * \star \star p<0.001\right)$ 

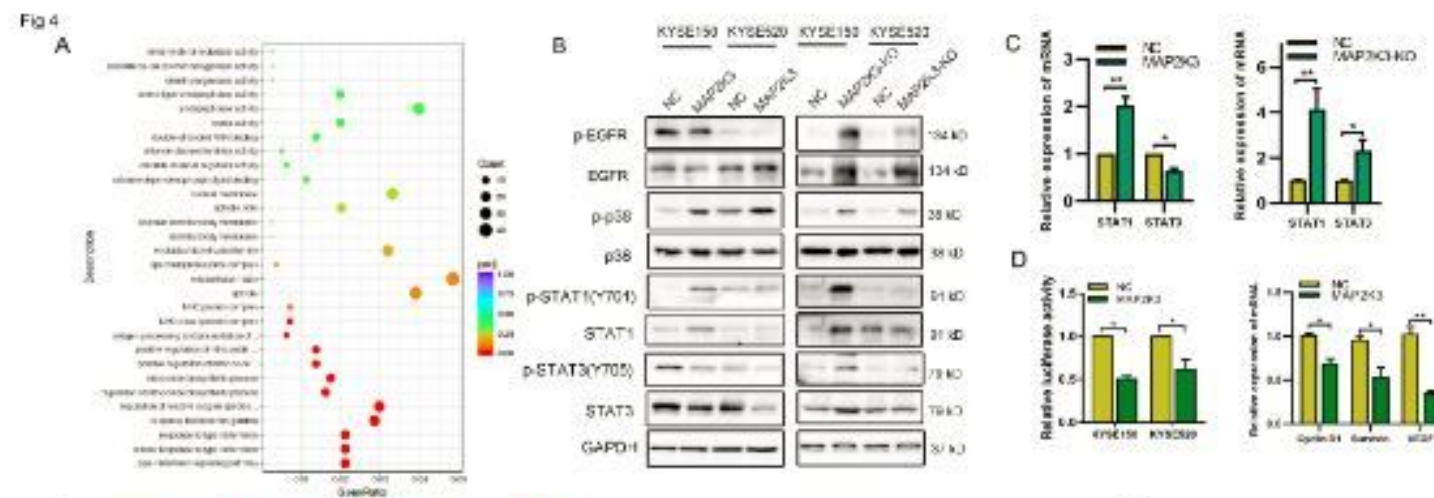

D
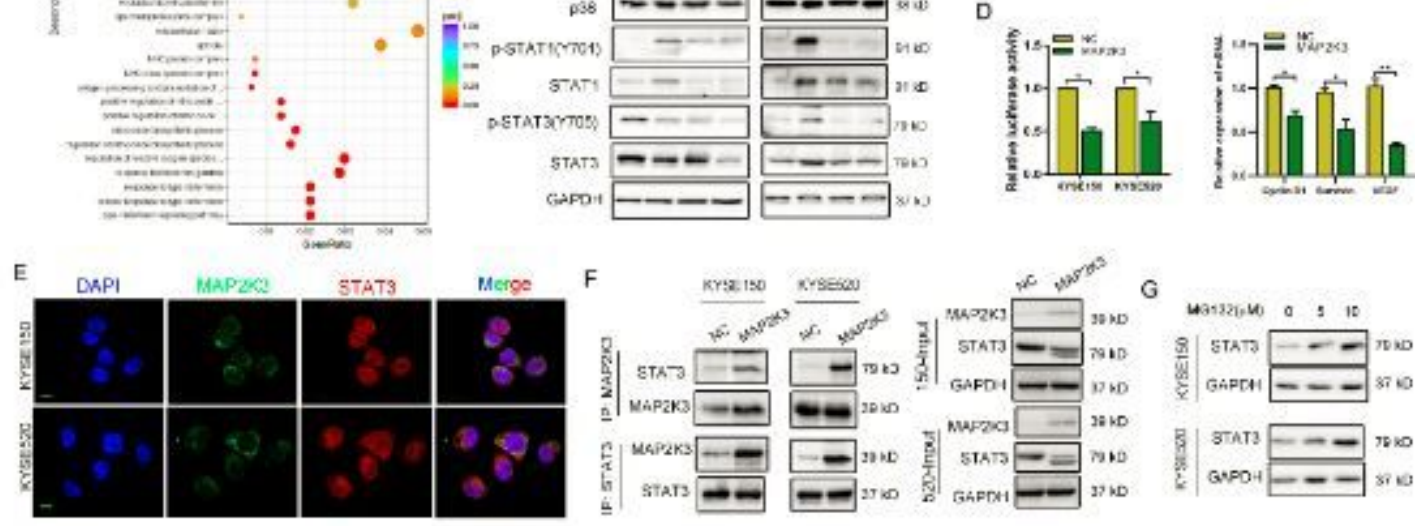

H
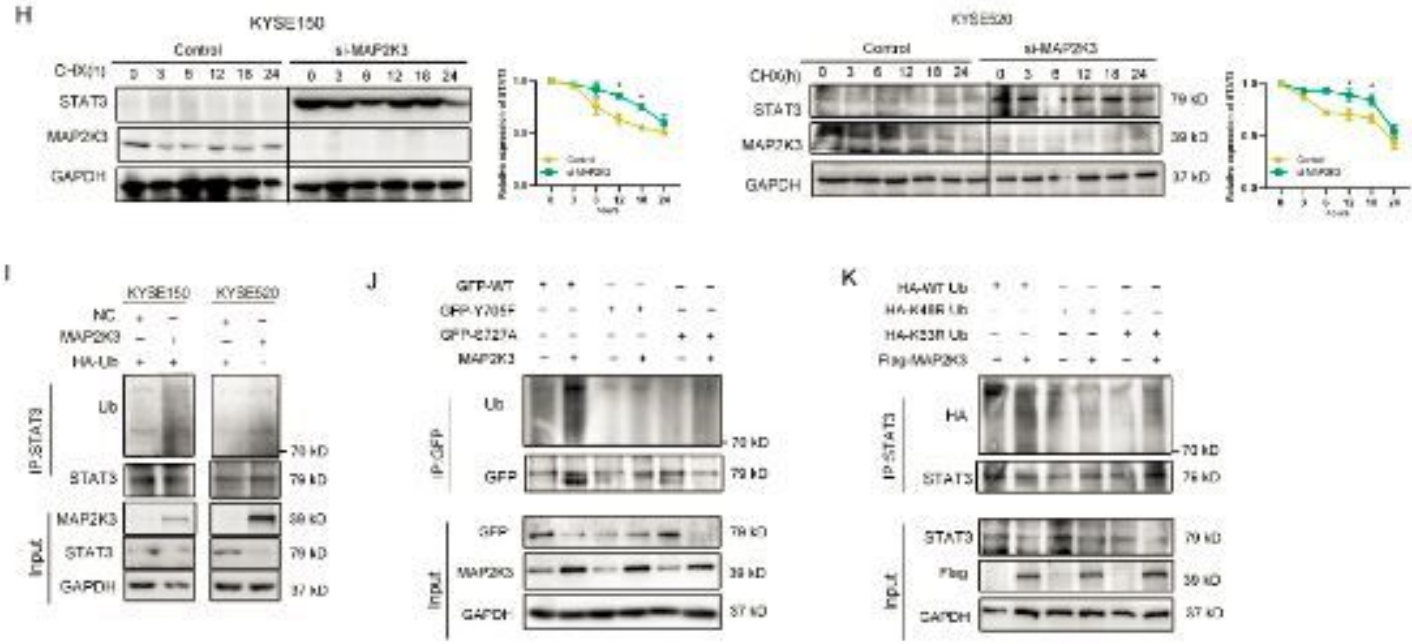

Figure 4

MAP2K3 modulates the EGFR/STAT3 signaling pathway in ESCC by promoting its proteasome degradation. A. Distribution of the top 20 enriched $\mathrm{GO}$ terms in biology process, cellular component and molecular function for the differentially expressed genes in MAP2K3-overexpressing ESCC cells based on RNA-seq analysis. B. Immunoblot analysis to detect phosphorylation and total EGFR, p38, STAT1 and STAT3 protein expression in ESCC cells. C. QPCR analysis to detect STAT1 and STAT3 RNA expression after MAP2K3 knockout and transfection in KYSE520 cells. D. The transcription activity of STAT3 was detected after MAP2K3 transfection by luciferase reporter assay (left panel). The mRNA expression of STAT3 downstream genes were detected by qRT-PCR (right panel). E. Co-localization of STAT3 and MAP2K3 was detected by immunofluorescence in KYSE150 and KYSE520 (Scale bar: $20 \mathrm{Im}$ ). F. Binding of endogenous MAP2K3 with STAT3 was detected by co-immunoprecipitation in KYSE150 and KYSE520 cells. G. Expression of STAT3 was detected by western blot after different doses of MG132 treatment for $24 \mathrm{~h}$. H. ESCC cells were treated by cycloheximide (CHX, $200 \mu \mathrm{g} / \mathrm{ml})$ in a time dependent manner after transfecting si-MAP2K3 and control. I. Immunoprecipitation to evaluate the level of ubiquitination of STAT3 in KYSE150 cells with MAP2K3 or control transfection. STAT3 ubiquitination was detected by 
immunoprecipitation with anti-STAT3 antibody and immunoblotting with anti-Ub antibody. J. GFP-STAT3 (WT), GFP-STAT3 (Y705F), or GFP-STAT3 (S727A) were transfected into KYSE150 cells together with the MAP2K3 plasmid or control. STAT3 ubiquitination was detected by immunoprecipitation with anti-GFP antibody and immunoblotting with anti-Ub antibody. K. HA tagged wide type, K48R and K63R Ub were transfected into KYSE150 cells together with the MAP2K3 plasmid. STAT3 ubiquitination was detected by immunoprecipitation with anti-STAT3 antibody and immunoblotting with anti-HA antibody. Error bars represent the SD from at least three independent biological replicates. $\left({ }^{\star} p<0.05 ;{ }^{* \star} p<0.01 ; \star \star \star p<0.001\right)$

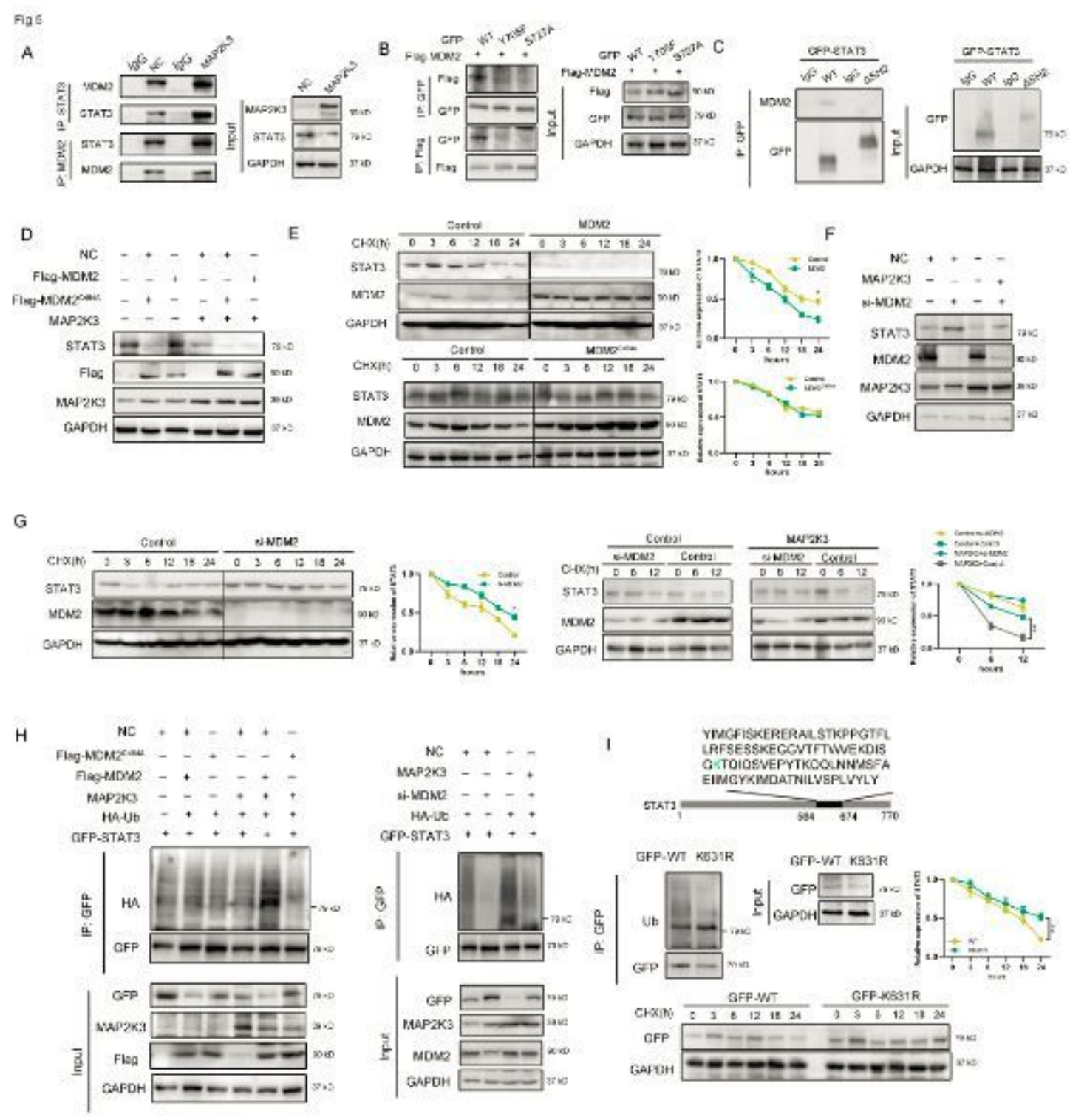

Figure 5

MAP2K3 interacted with E3 ligase MDM2 to promote STAT3 degradation. A. The interaction of STAT3, MAP2K3 and MDM5 was detected by co-immunoprecipitation in KYSE150 cells. B. The interaction of GFP tagged wide type, Y705F, S727A STAT3 and Flag-MDM2 were detected by co-immunoprecipitation in 293 T cells. C. The interaction of GFP tagged wide type STAT3 or SH2 domain deletion STAT3 and MDM2 were detected by co-immunoprecipitation in KYSE150 cells. D. MDM5 decreased STAT3 protein. KYSE150 cells were transfected with Flag-MDM5 or Flag-MDM5C464A as well as control or MAP2K3 transfection. 
The protein expression level of STAT3 was assayed by western blot. E. The cells expressing wide type MDM5 or MDM5C464A were treated with cycloheximide (CHX, $200 \mu \mathrm{g} / \mathrm{ml})$. The protein levels of STAT3 and MDM5 were analyzed by western blot. F. Knockdown MDM5 increased STAT3 protein. KYSE150 cells were transfected with si-MDM5 as well as control or MAP2K3 transfection. G. KYSE150 cells were transfected with control or MDM5 siRNAs treated with CHX, the protein levels of STAT3 and MDM5 were analyzed by western blot. $\mathrm{H}$. MDM5 ubiquitylates STAT3. KYSE150 cells were transfected with indicated plasmids or siRNA for $48 \mathrm{~h}$. Cell lysates were immunoprecipitated with anti-GFP and analyzed by immunoblotting with indicated antibodies. I. KYSE150 cells were transfected with indicated plasmid. Lysates were immunoprecipitated with anti-GFP, and western blots were performed to analyze the presence of indicated proteins and levels of ubiquitination. The protein level of STAT3 was quantitated by Image $\mathrm{J}$ and normalized to GAPDH levels. Error bars represent the SD from at least three independent biological replicates. ( ${ }^{\star} p<0.05 ;{ }^{* *} p<0.01 ; * * * p<0.001$ )

tose

A

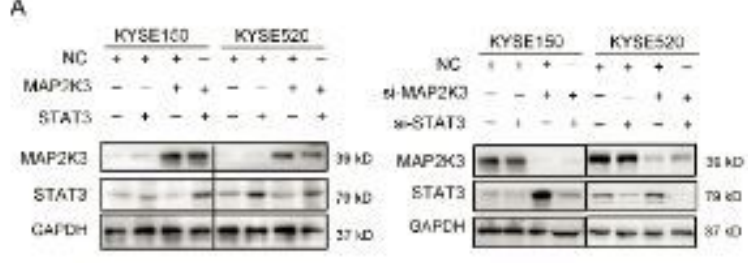

c

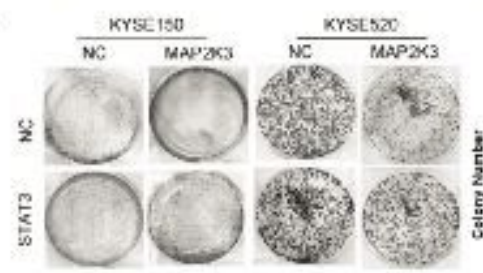

D
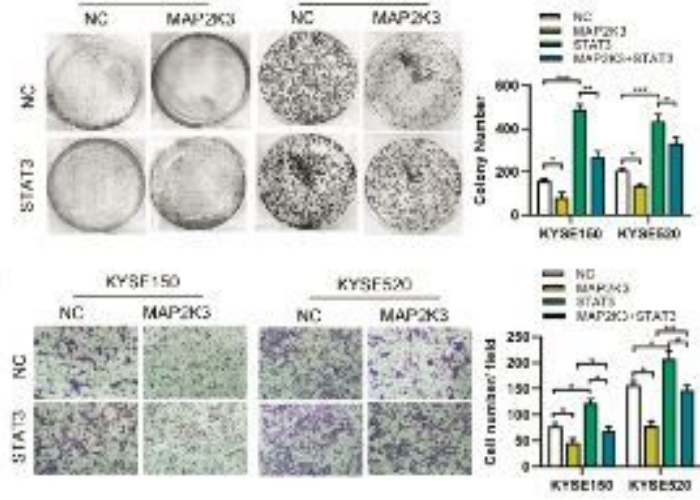

B

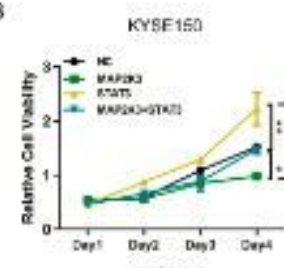

KYSE 160
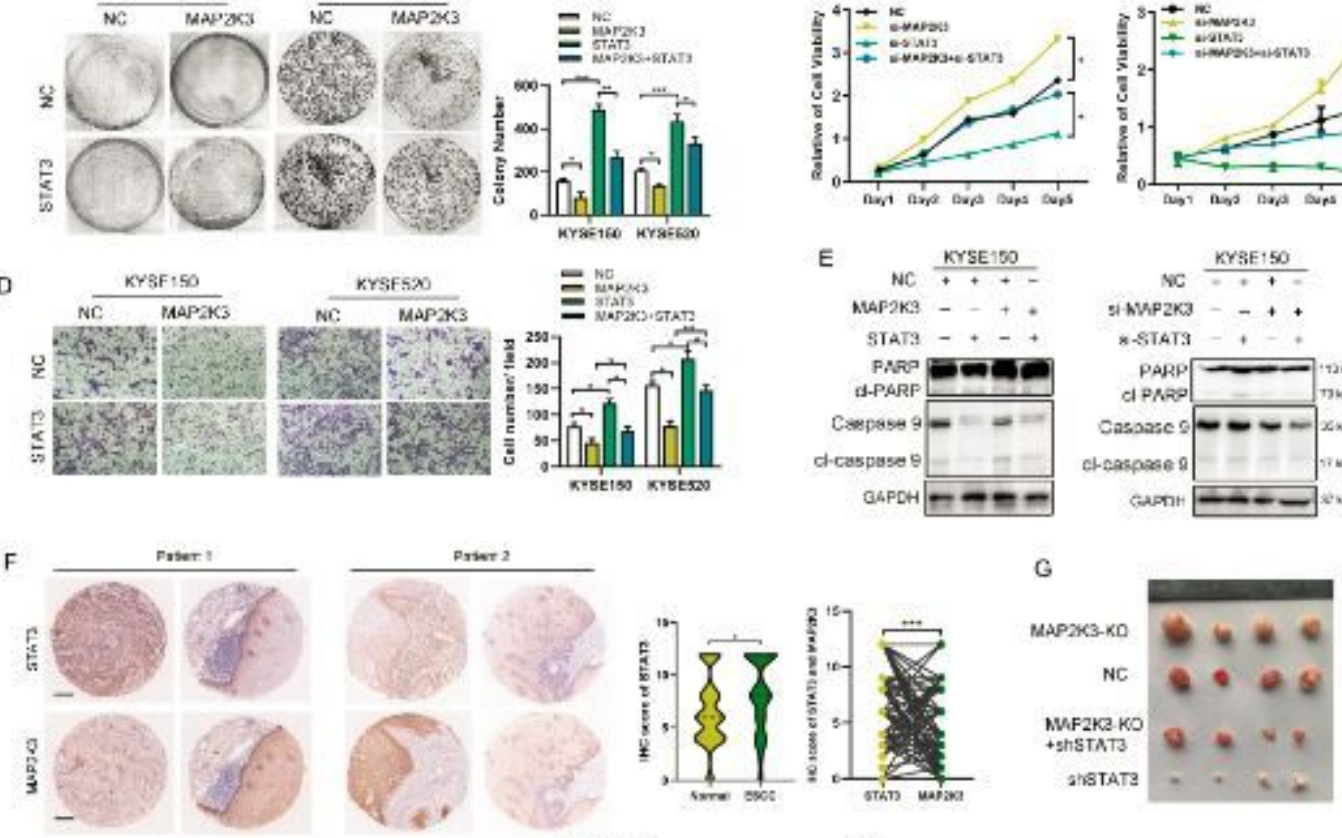

$\mathrm{H}$
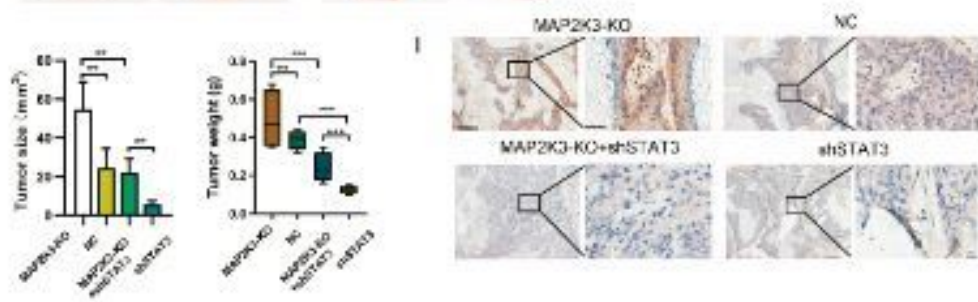
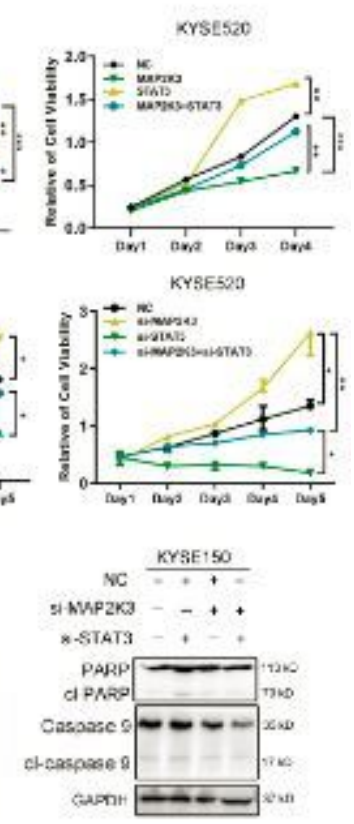

KYSEST
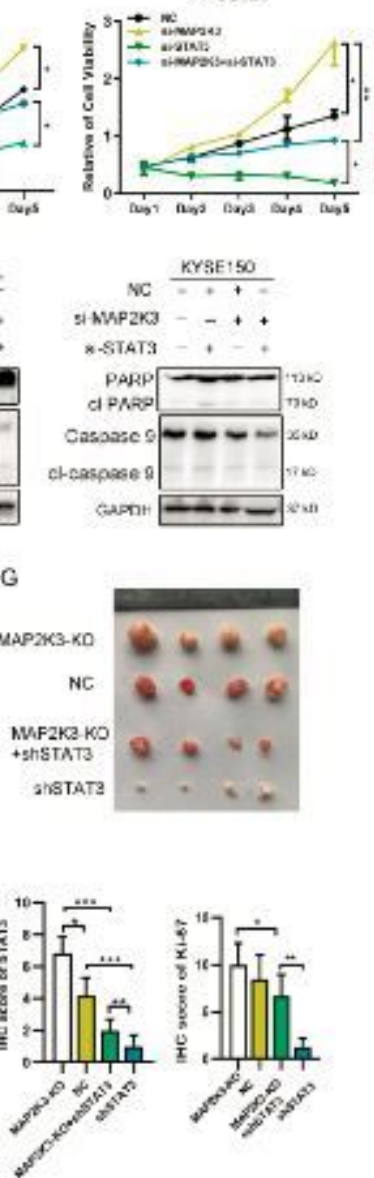

Figure 6 
STAT3 is an essential factor in MAP2K3-mediated tumorigenesis in ESCC. A. The transfection efficiency of indicated plasmids were detected by westernblot. B. Cell growth of KYSE150 and KYSE520 cells after STAT3 or si-STAT3 and MAP2K3 or si-MAP2K3 transfection was detected by CKK8. C. Colony formation was detected in STAT3 and MAP2K3 transfection cells. D. Cell invasion was detected in STAT3 and MAP2K3 transfection cells by transwell assay. E. Western blot to detect expression of PARP and caspase 9 in KYSE150 cells after STAT3 or si-STAT3 and MAP2K3 or si-MAP2K3 transfection. F. MAP2K3 and STAT3 expression were evaluated by IHC ( $n=140$, Scale bars: $200 \mathrm{Im})$. Correlations between STAT3 and MAP2K3 expression levels in ESCC patients. G. The 5-week-old immunodeficient nude mice (4 mice per group) were injected subcutaneously with indicated cells $(1 \times 107$ cells). $\mathrm{H}$. Tumor volume and weight was measured at day 28. I. Immunohistochemistry analysis of STAT3 and Ki67 of tumor xenografts with indicated treatment (Scale bar, $50 \mu \mathrm{m}, 200 \mu \mathrm{m})$. Error bars represent the SD from at least three independent biological replicates $\left({ }^{\star} p<0.05 ;{ }^{* \star} p<0.01\right.$; $\left.{ }^{* \star *} p<0.001\right)$ Fiq 7

A

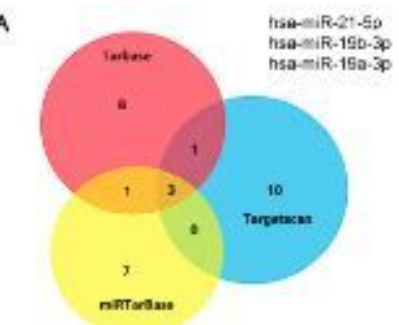

c

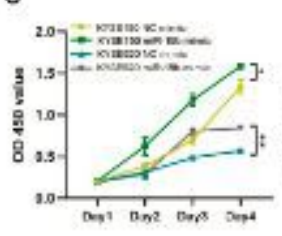

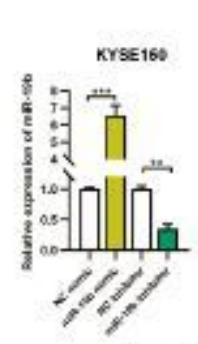

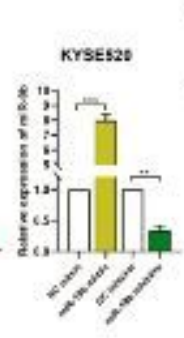

D Mc rags
B
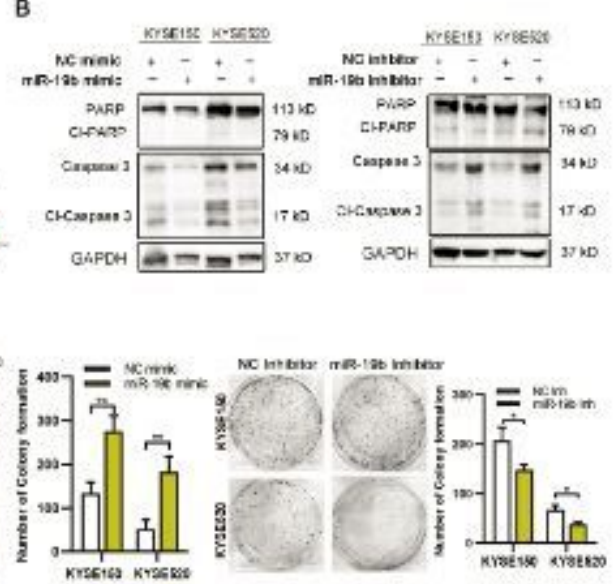

Krestis KrgaWs

E
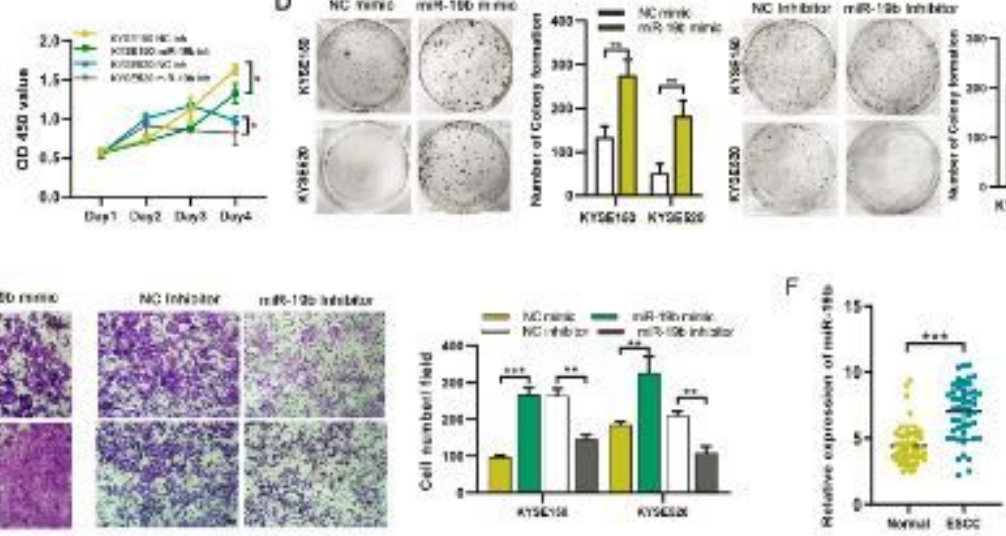

G
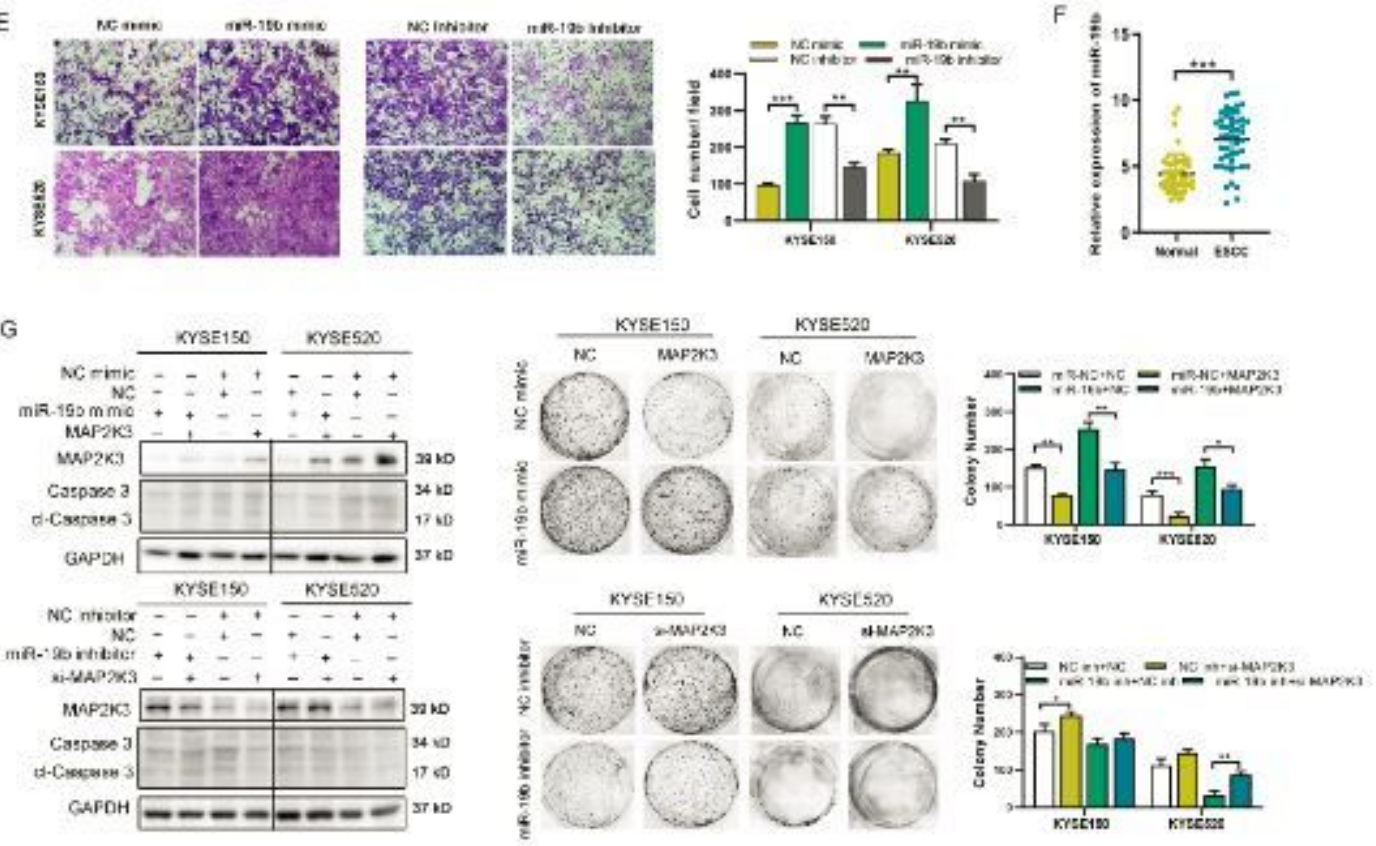

Figure 7 
MiR-19b-3p mediated cell proliferation and invasion via suppressing MAP2K3. A. Venn Diagram: number of predicted miRNAs from Targetscan, DIANA-TarBase and miRTarBase is shown, identifying three miRNAs: miR-21-5p, miR-19a-3p and miR-19b-3p. The transfection efficiency was detected after miR-19b$3 p$ mimic or inhibitor transfection. B. Western blot for apoptosis biomarkers, PARP and caspase 3 after miR-19b-3p mimic transfection. C.CCK8 assay was performed to detect the cell proliferation in KYSE150 cells transfected with miR-19b mimic, miR-19b inhibitor or control vector. D. Colony formation of KYSE150 and KYSE520 cells transfected with miR-19b mimic, miR-19b inhibitor or control vector was detected. E. The cell invasion ability was detected by transwell assay in KYSE150 and KYSE520 cells transfected with miR-19b mimic, miR-19b inhibitor or control vector. F. The expression of miR-19b-3p was detected in ESCC tissues and case-matched normal esophageal epithelial(n=48). G. Colony formation analyses in KYSE150 cells transfected with miR-19b-3p plus MAP2K3 plasmid transfection. Error bars represent the SD from at least three independent biological replicates. ( ${ }^{\star} p<0.05 ;{ }^{* \star} p<0.01$; $\left.{ }^{\star \star \star} p<0.001\right)$.

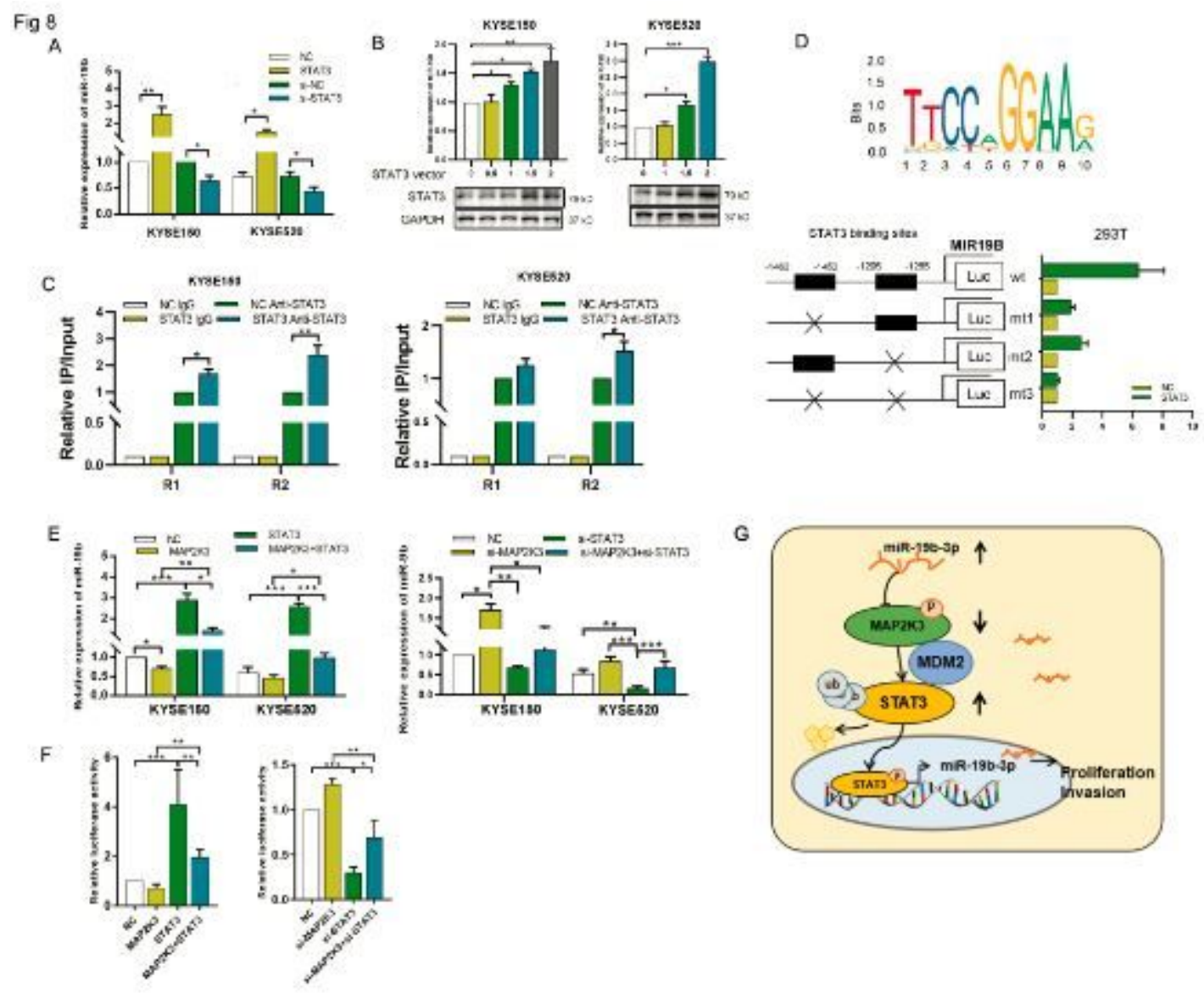

Figure 8

STAT3 binds with miR-19-3p promoter to increase miR-19-3p expression. A. Expression of miR-19b-3p was detected in STAT3 overexpressed and knockdown KYSE150 and KYSE520 cells. B. Expression of miR-19b-3p was detected in different dose of STAT3 plasmids transfected KYSE150 and KYSE520 cells. C. ChIP assay was performed to detect the binding of STAT3 in the promoter of MIR19 after STAT3 transfection. D. The binding sequence of STAT3 (upper panel). The 293T cells were co-transfected with different combinations of wild type (wt) and mutated reporter constructs (mt1, mt2 or mt3), STAT3 and 
control. The relative luciferase activity was analyzed as previously described (lower panel). E. Expression of miR-19b-3p was detected in KYSE150 and KYSE520 cells with indicated transfection. F. The relative luciferase activity of MIR19B was analyzed in the indicated transfected KYSE150 and KYSE520 cells. Error bars represent the SD from at least three independent biological replicates. $\left({ }^{*} p<0.05 ;{ }^{* \star} p<0.01\right.$; $\star \star \star p<0.001)$

\section{Supplementary Files}

This is a list of supplementary files associated with this preprint. Click to download.

- sUPPLEMENTALfigure6MIR19bMAP2K3.tif

- sUPPLEMENTALfigure6MIR19bMAP2K3.tif

- SupplementalFigure5stat3.tif

- SupplementalFigure5stat3.tif

- SupplementalFigure4.tif

- SupplementalFigure4.tif

- renamed1efb0.tif

- renamed1efb0.tif

- renamed18e92.tif

- renamed18e92.tif

- SupplementalFIGUR1.tif

- SupplementalFIGUR1.tif

- supplementalprimermkk3.docx

- supplementalprimermkk3.docx 